\title{
Three-Dimensional Ageostrophic Motion in Mesoscale Vortex Dipoles
}

\author{
EnRic Pallàs-Sanz And Álvaro Viúdez \\ Institut de Ciències del Mar, Barcelona, Spain
}

(Manuscript received 23 September 2005, in final form 12 May 2006)

\begin{abstract}
The three-dimensional motion of mesoscale baroclinic dipoles is simulated using a nonhydrostatic Boussinesq numerical model. The initial conditions are two ellipsoidal vortices of positive and negative potential vorticity anomalies. The flow is moderately ageostrophic with a maximum absolute Rossby number equal to 0.71 . The trajectory of the dipole is related to the maximum potential vorticity anomaly and size of the vortices. Three cases are considered depending on the curvature of the dipole trajectory: negative, close to zero, and positive. The ageostrophic flow strongly depends on the distance between the ellipsoidal vortices $d_{0}$. For small $d_{0}$ the vortices move steadily as a compact dipole, and the vertical velocity $w$ has an octupolar three-dimensional pattern. The horizontal ageostrophic velocity is due to the advective acceleration of the flow, particularly the centripetal acceleration. The speed acceleration is only relatively important at the rear and front parts of the dipole axis, where the flow curvature is small but where the flow confluence and diffluence are, respectively, large. The geostrophy is maximal at the dipole center, on the dipole axis, where both curvature and speed acceleration are minimal. As $d_{0}$ increases, the dipole self-propagating velocity and the extreme values of $|w|$ decrease, and vortex oscillations highly distort the octupolar pattern of $w$. In all cases, as is typical of balanced mesoscale geophysical flows, the vertical velocity is related to the advection of vertical vorticity by the horizontal shear velocity $\mathbf{u}_{h z} \cdot \nabla_{h} \zeta$.
\end{abstract}

\section{Introduction}

Mesoscale dipole eddies are flow structures consisting of two patches of oppositely signed vertical vorticity (Ikeda et al. 1984; Ginzburg and Fedorov 1984; Fedorov and Ginsburg 1986). These dipole eddies, or mushroom-like currents, are unsteady coherent phenomena, which are found widespread in both the oceans and atmosphere. They have been the subject of extensive observational, theoretical, laboratory, and numerical research. For example, mesoscale dipoles have been repeatedly observed from satellite imagery in surface coastal currents (Millot 1985; Fedorov and Ginsburg 1986; Ahlnäs et al. 1987; Johannessen et al. 1989), and recently from a hydrographic cruise south of Madagascar (de Ruijter et al. 2004).

Laboratory experiments have reproduced quasi-twodimensional dipoles when a jet is applied to a volume fluid with some vertical motion constraint, typically 1) stable stratification (van Heijst and Flór 1989; Voro-

Corresponding author address: Enric Pallàs-Sanz, Institut de Ciències del Mar, P. Marítim de la Barceloneta 37-49, 08003 Barcelona, Spain.

E-mail: epallas@cmima.csic.es payev et al. 1991; Flór and van Heijst 1994), 2) rotation in a homogeneous fluid (Flierl et al. 1983; Fedorov and Ginsburg 1986), or 3) both rotation and stable stratification (Voropayev et al. 1997). Other processes involving dipole generation are the development of centrifugal instability of a barotropic anticyclonic vortex in a rotating fluid (Kloosterziel and van Heijst 1991; Orlandi and Carnevale 1999) and the motion of a vertical cylinder through a rotating fluid in a sloping bottom tank (Fuentes and van Heijst 1994; Sansón et al. 2001).

Numerical models have successfully simulated the oceanic dipole formation through the instability of currents. An initially unstable barotropic jet on the $\beta$ plane splits up into dipoles that migrate following a curved trajectory (Flierl et al. 1987). Shimada and Kubokawa (1997), using the contour dynamics method, simulated an unstable boundary current evolving into barotropic dipoles that transported the trapped coastal water mass away from the boundary. The interaction between detached rings and near-field circulation of a baroclinic meandering jet produces isolated dipolar eddies, which propagate with a path curvature that depends on the strength of the vorticity poles (Flierl et al. 1983; Robinson et al. 1988; Bush et al. 1996; Spall and Robinson 1990; Spall 1995). Similar results on the dipole trajec-

DOI: $10.1175 / \mathrm{JPO} 2978.1$ 
tory have been recently obtained in quasigeostrophic numerical simulations of the interaction between a stable zonal jet and a vortex (Vandermeirsch et al. 2003a,b).

Analytical solutions of the two-dimensional Euler equation for dipoles were given by Meleshko and van Heijst (1994) and Lamb (1932). Form-preserving, uniformly translating vortex dipoles solutions, or modons (Stern 1975), have been extensively studied in layer models. A modon consists of two separated uniform vorticity cores with a relationship between streamfunction and vorticity within the vortex core that can be linear (e.g., Lamb 1932; Stern 1975; Flierl et al. 1980; Kizner 1997) or nonlinear (McWilliams 1983; Morel and McWilliams 1997; Kizner et al. 2003; Khvoles et al. 2005).

Mesoscale vortex dipoles are also important because of their role in the mixing processes and transport of water properties in the ocean. Their ability to transport heat or other scalar quantities was addressed, in a twolayer flow, by Hogg and Stommel (1985), who named these baroclinic dipoles hetons. Analytical solutions for a two-layer geophysical heton and the coalescence of geostrophic vortices have been obtained by Pedlosky (1985) and Griffiths and Hopfinger (1987), respectively. More recently, Gryanik et al. (2000) developed an analytical theory, using the quasigeostrophic form of the potential vorticity (PV) evolution equation, for the dynamics of a population of three-dimensional hetons in a fast-rotating stratified fluid. Point-vortex dynamics (Batchelor 1967, section 2.6) was used to address the volume transport by the dipole propagation (Flierl 1987), a feature also observed experimentally in stratified fluids (Voropayev et al. 1991; Flór and van Heijst 1994). The mass exchange and chaotic particle transport by a dipole in the $\beta$ plane is related to the change of vertical vorticity $\zeta$ and potential vorticity conservation in the vortices (Fuentes et al. 1995), where the main transport mechanism is the fluid entrainment and detrainment from the vortices interior (Eames and Flór 1998). Unsteady dipoles in a balanced flow may also produce the spontaneous generation of inertia-gravity waves. This process has been observed experimentally in unsteady vortex collisions (Afanasyev 2003).

Most of the research mentioned above has dealt with the two-dimensional (2D) characteristics of the dipolar vortex, which is consistent as a first approximation to mesoscale geophysical dynamics. Beyond the 2D approximation mesoscale oceanic dipoles are inherently three-dimensional (3D) structures. For example, the mesoscale upwelling associated to these coherent structures contributes significantly to the biological productivity increasing the nutrients and phytoplankton concentrations in the euphotic zone (e.g., Strass 1992; Flierl and Davis 1993; Robinson et al. 1993; McGillicuddy et al. 1998). The aim of this paper is to describe, using numerical simulations, the full 3D ageostrophic motion of these geophysical dipolar vortices.

The analysis of the relatively small 3D ageostrophic motion of inherently ageostrophic structures as mesoscale dipoles requires two important conditions, namely, (i) the dynamical equations must be precise and diffusionless enough to reproduce the small balanced vertical velocity field in the dipole along its translation and (ii) the numerical initialization technique must prevent the generation of inertia-gravity waves due to the initial imbalance between density and velocity fields (Hoskins et al. 1985; Ford et al. 2000; Mohebalhojeh and Dritschel 2000; Mohebalhojeh and Dritschel 2001; Viúdez and Dritschel 2004a). Quasigeostrophic (e.g., Daley 1991, section 7) or semigeostrophic dynamics prohibit this generation but they may filter an important part of the balanced 3D flow as well (Viúdez and Dritschel 2004b). The numerical model, initialization, and initial conditions are described in section 2 . Here we use a 3D nonhydrostatic Boussinesq numerical model (Dritschel and Viúdez 2003, hereinafter DV03) initialized using the PV initialization approach (Viúdez and Dritschel 2003, hereinafter VD03). The PV is represented by contours lying on isopycnal surfaces and is explicitly conserved so that the numerical diffusion is very small. The PV initialization approach is based in the slow increase, during the initialization period, of the prescribed PV in every fluid particle, in a Lagrangian way. This method avoids, for sufficiently large initialization periods, the initial generation of inertia-gravity waves.

Section 3 describes the numerical results. We select three classes of numerical simulations depending on the amount of PV in each vortex. These correspond to dipole trajectories with negative, close to zero, and positive curvature. The negative-curvature case is used as the reference simulation and explained with more detail. While the 3D vertical velocity pattern of isolated vortices is octupolar, it is found that the vertical velocity in the pair of vortices forming the dipole has an octupolar structure as well, as long as the initial distance between vortices is close enough. Vortex-vortex interactions inside the dipole may however eventually break this octupolar structure and originate more complex patterns.

\section{The numerical model and initial conditions}

\section{a. The numerical model}

We use a 3D triply periodic nonhydrostatic model to simulate rotating, inviscid, stratified, and volume-pre- 
serving flows under the Boussinesq and $f$-plane approximations. The model conserves explicitly the potential vorticity $\Pi$ on a contour representation on isopycnals using the contour-advective semi-Lagrangian algorithm (Dritschel and Ambaum 1997). The state variables are the components of the vector potential $\boldsymbol{\varphi}=(\varphi, \psi, \phi)$, which provide the 3D velocity $\mathbf{u}$ and the vertical displacement of isopycnals $\mathcal{D}$ :

$$
\mathbf{u}=-f \boldsymbol{\nabla} \times \boldsymbol{\varphi} \quad \text { and } \quad \mathcal{D}=-\varepsilon^{2} \boldsymbol{\nabla} \cdot \boldsymbol{\varphi}
$$

where $\varepsilon=1 / c=f / N$ is the ratio between the constant Coriolis and the mean Brunt-Väisälä frequencies. The three prognostic equations are (i), (ii) the equations for the rate of change of the horizontal ageostrophic dimensionless vorticity $\mathbf{A}_{h}=(\mathcal{A}, \mathcal{B}) \equiv \boldsymbol{\omega}_{h}^{\prime} / f \equiv\left(\boldsymbol{\omega}_{h}-\boldsymbol{\omega}_{h}^{g}\right) / f$, where $\boldsymbol{\omega}_{h}$ and $\boldsymbol{\omega}_{h}^{g}$ are the total and geostrophic horizontal vorticity, respectively; and (iii) the explicit material conservation of PV anomaly $\varpi$ on isopycnals. The horizontal components of the vector potential $\varphi_{h}=(\varphi$, $\psi)$ are recovered every time step from the inversion of $\mathbf{A}_{h}=\nabla^{2} \boldsymbol{\varphi}_{h}$, while the vertical potential $\phi$ is obtained from the inversion of the definition of the dimensionless PV anomaly $\varpi \equiv 1-\Pi \equiv 1-(\boldsymbol{\omega} / f+\mathbf{k}) \cdot(\mathbf{k}-\boldsymbol{\nabla} \mathcal{D})$. More details are given in DV03 and VD03.

\section{b. Model parameters}

In the reference simulation we use a $64^{3}$ grid with 64 isopycnals in a domain of vertical extent $L_{Z}=2 \pi$ (which defines the unit of length) and horizontal extents $L_{X}=L_{Y}=c L_{Z}$, with $c \equiv N / f=100$ as the ratio of the mean Brunt-Väisälä to Coriolis frequency. We take the (mean) buoyancy period (b.p.) as the unit of time by setting $N=2 \pi$. Thus, one inertial period (i.p.) equals 100 b.p.

To avoid the generation of grid-scale noise, a biharmonic hyperdiffusion term $-\mu\left(\nabla_{q}^{4} \mathcal{A}, \nabla_{q}^{4} \mathcal{B}\right)$, where $\nabla_{q} \chi \equiv(\partial \chi / \partial x, \partial \chi / \partial y, \varepsilon \partial \chi / \partial z)$ is the gradient operator in the vertically stretched space, is added to the equations for the rate of change of $\mathbf{A}_{h}$. The hyperviscosity coefficient $\mu$ is chosen by specifying the damping rate ( $e$ folding, $e_{f}$ ) of the largest wavenumber in spectral space per inertial period, which was set constant to 10 .

The other parameters are the time step $\delta t=0.1$ b.p., and the initialization time $\Delta t_{I}=5$ i.p. The initialization time is the minimum time required for the fluid to reach its initial perturbed state with minimal generation of inertia-gravity waves. The total period of simulation is $t_{F}=200$ i.p.

\section{c. Initial conditions}

The 3D dipoles are simulated as two equal ellipsoids of oppositely signed PV anomaly. The initial PV con-

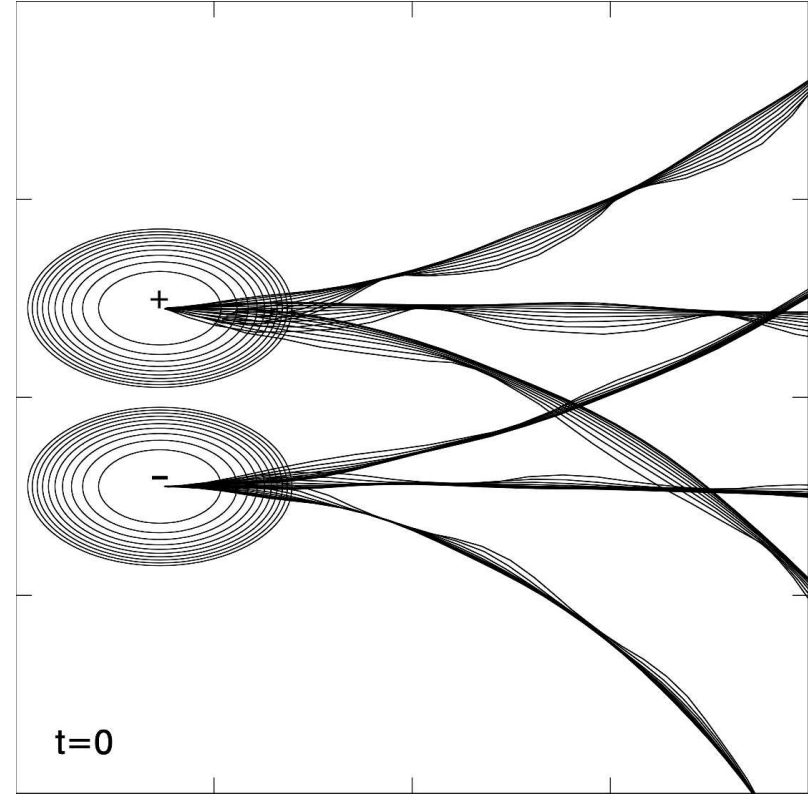

FIG. 1. Initial PV contours in the middle isopycnal $l=32$. Horizontal domain is $x, y \in[-\pi, \pi] c$. The vortex trajectories for the three cases (positive, close to zero, and negative curvature) at the layers $i_{Z} \in[24,32]$ are also included. The trajectories of the deeper part of the vortices experience oscillations seen as departures from the constant curvature observed in the middle layer.

tours, identical in the three cases, are shown in Fig. 1. The dipole, with the cyclone located in the north and the anticyclone in the south, is initially placed in the western side of the domain since an eastward selfpropagation is expected. The number of initial PV contours in the middle isopycnal $(l=32)$ in every vortex is $n_{c}=10$, varying from $\varpi \cong 0$ on the outermost surface to extreme PV anomaly $\varpi=\varpi_{0}^{ \pm}$at the core, where $\varpi_{0}^{+}$and $\varpi_{0}^{-}$are the maximum and minimum PV anomalies in the cyclone and anticyclone, respectively.

The PV jumps, that is, the PV increment across each PV contour, differ in the ellipses and depend on the magnitude of $\varpi_{0}^{ \pm}$. Therefore the PV anomaly increment is fixed for all contours $\Delta \varpi=\varpi / n_{c}$ (exception is the outermost contour where $\Delta \varpi / 2$ ). The outermost PV ellipsoidal layer has horizontal major, minor, and vertical semiaxes $\left(a_{M}, a_{m}, a_{Z}\right)=\pi(c / 3, c / 5,1 / 3)$. The initial distance between the vortex centers is $d_{0}=\sqrt{2 c}$. Further details about the configuration of a single ellipsoid are given in VD03. Henceforth, a vortex core refers to the fluid with extreme constant values of $\varpi$; that is, $\varpi \in$ $\left[n_{c}-1, n_{c}\right] \varpi_{0}^{ \pm} / n_{c}$. For a fixed vortex volume, defined by $\left(a_{M}, a_{m}, a_{Z}\right)$, the trajectory curvature of the dipole $\kappa_{d}$ depends on the two values $\varpi_{0}^{ \pm}$. The next section describes the numerical results for three cases: $\kappa_{d}<0$, $\kappa_{d} \cong 0$, and $\kappa_{d}>0$. 


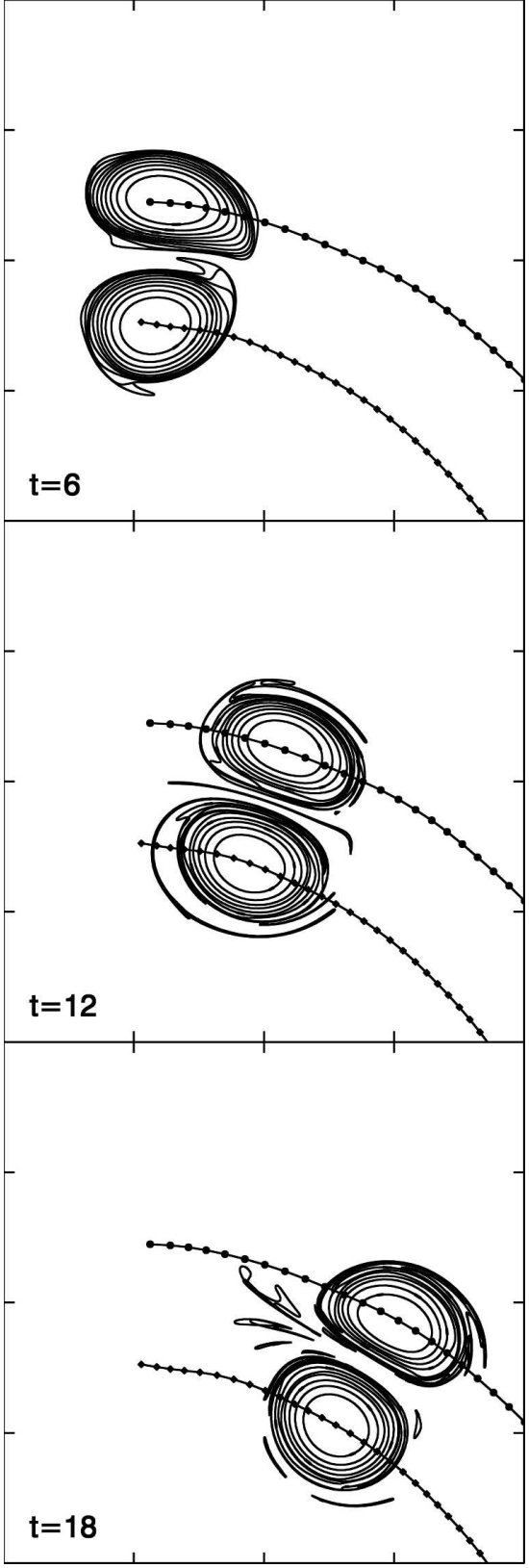

FIG. 2. Time evolution at $t=6,12$, and 18 i.p. of the PV contours in the middle isopycnal for the case $\kappa_{d}<0\left(\varpi_{0}^{ \pm}= \pm 0.75\right)$. Horizontal domain is $x, y \in[-\pi, \pi] c$. The vortex trajectories, computed every inertial period and starting at $t=5$ i.p. (black symbols), are included.

\section{Numerical results}

\section{a. Potential vorticity}

1) CASE $\kappa_{d}<0$

In this case $\varpi_{0}^{+}=-\varpi_{0}^{-}=0.75$ (Fig. 2). The PV anomalies cause a moderately large ageostrophic flow both inertial and statically stable. The time average of the extreme Rossby numbers in the domain $\mathcal{R}^{ \pm} \equiv$ $\pm\langle\max \{ \pm \zeta(\mathbf{x}, t)\}\rangle / f$, where $\zeta$ is the vertical vorticity and the time average \langle\rangle is from $t=5$ to $t=30$ i.p., are $\left\{\mathcal{R}^{-}\right.$, $\left.\mathcal{R}^{+}\right\}=\{-0.71,+0.52\}$. The time average of the maximum Froude number $\mathcal{F} \equiv\left\langle\max \left\{\left|\boldsymbol{\omega}_{h}(\mathbf{x}, t)\right|\right\}\right\rangle / N=0.27$.

The PV vortices soon deform from their initial elliptical configuration to a more steady shape and start their eastward propagation (Fig. $2, t=6,12$ i.p.). Some PV filaments from the outermost PV layers are expelled back from the dipole axis at later times (Fig. 2, $t=18$ i.p.), but the vortices remain close from each other and propagate together as a compact dipolar structure. There is also a clear vortex asymmetry with the cyclone being more elliptical than the anticyclone (Fig. 2, $t=18$ i.p.).

Because of the small numerical diffusivity of the algorithm the lifetime of the dipoles is very long. Most of simulations were carried out until $t_{F}=200$ i.p., though only results from the first 30 i.p. are described here. Diabatic effects and eddy diffusion do, however, significantly contribute to the dynamical decay of these structures on large time scales (Morel and McWilliams 1997).

\section{2) CASES $\kappa_{d} \cong 0$ AND $\kappa_{d}>0$}

In these cases the PV anomaly of the cyclone is increased to $\varpi_{0}^{+}=+0.95$ (case $\kappa_{d} \cong 0$ ) and $\varpi_{0}^{+}=+1.15$ (case $\kappa_{d}>0$, i.e., approximately $25 \%$ and $50 \%$ with respect to their values in the reference simulation), while the PV anomaly of the anticyclone $\varpi_{0}^{-}=-0.75$ is unmodified.

The time-averaged Rossby number $\mathcal{R}^{+}$increases to 0.62 (case $\kappa_{d} \cong 0$ ) and 0.71 (case $\kappa_{d}>0$ ), due to the larger PV in the cyclone. The time-averaged Froude number $\mathcal{F}$ remains however close to the value of the reference simulation, with $\mathcal{F}=0.27$ (case $\kappa_{d} \cong 0$ ) and $\mathcal{F}=0.28\left(\right.$ case $\left.\kappa_{d}>0\right)$.

The PV contours for cases $\kappa_{d} \cong 0$ and $\kappa_{d}>0$ are shown in Figs. 3 and 4, respectively. The vortices bound together again and move eastward, peeling off filaments during their self-propagation (Fig. $3, t=12,18$ i.p.; Fig. $4, t=12,18$ i.p.). In the case $\kappa_{d} \cong 0$ the dipole remains more axisymmetric (Fig. 3, $t=18$ i.p., though this is more clearly seen at later times) than in both the reference case $\kappa_{d}<0$ (Fig. 2, $t=18$ i.p.) and the positive curvature case (Fig. 4, $t=18$ i.p.).

\section{b. Trajectories and curvatures}

Since the 3D vortices may be considered as a family of closed isosurfaces of $\varpi$, their position at any time can be defined as 


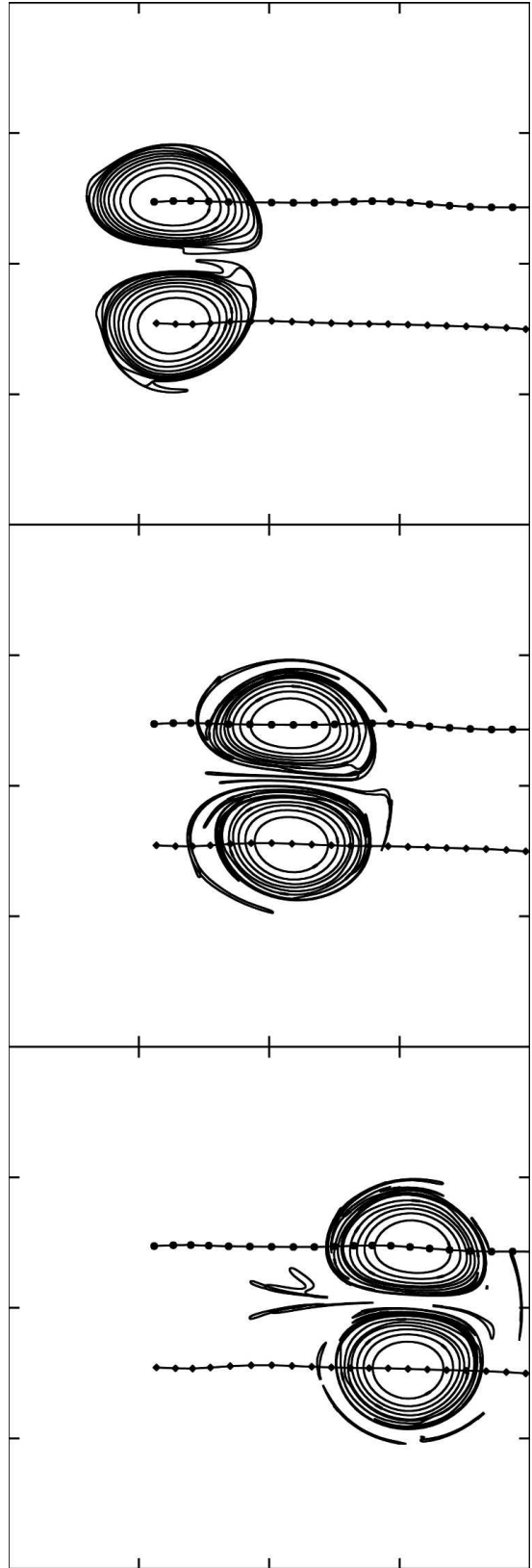

FIG. 3. As in Fig. 2 but for the case $\kappa_{d} \cong 0\left(\varpi_{0}^{+}=0.95\right.$ and $\left.\varpi_{0}^{-}=-0.75\right)$.

$$
\mathbf{R}^{ \pm}(t) \equiv \frac{\int_{\mathcal{V}^{ \pm}} \varpi(\mathbf{x}, t) \mathbf{r}(\mathbf{x}, t) d V}{\int_{\mathcal{V}^{ \pm}} \varpi(\mathbf{x}, t) d V},
$$

where $\mathcal{V}^{ \pm}$is the volume of the cyclone and anticyclone, and $\mathbf{r}$ is the position vector. The position of the dipole $\mathbf{R}_{d}(t)$ can be defined as the average:

$$
\mathbf{R}_{d} \equiv \frac{1}{2}\left(\mathbf{R}^{+}+\mathbf{R}^{-}\right)
$$

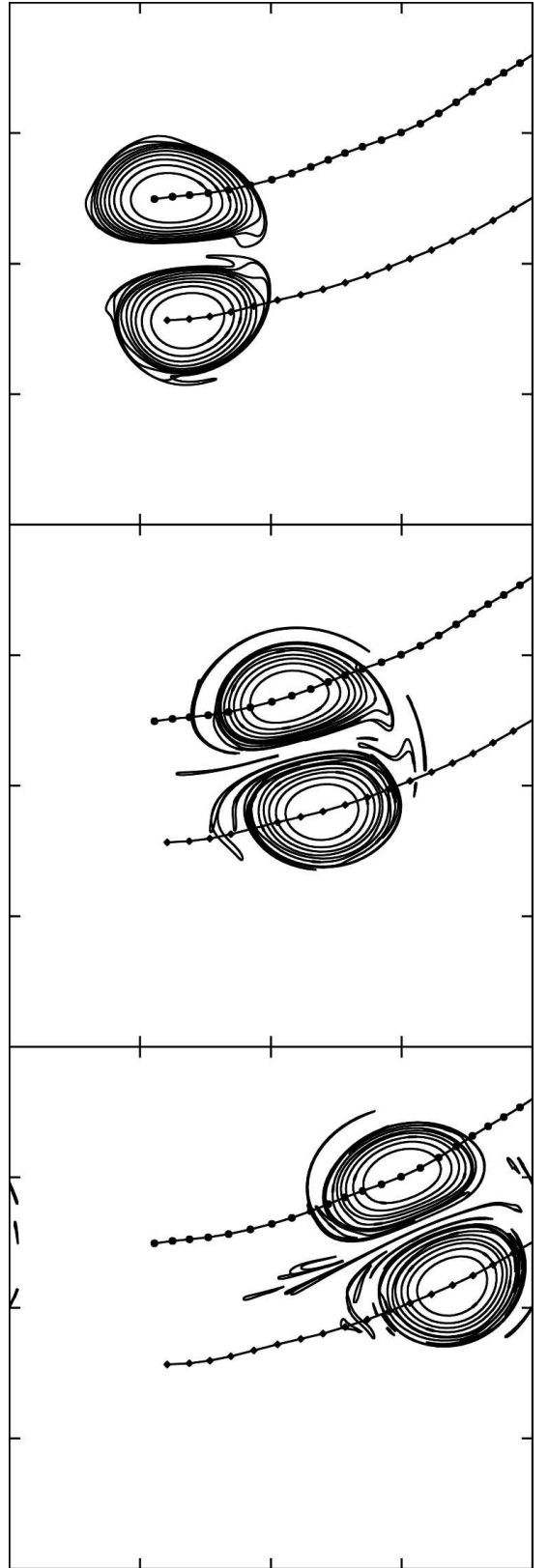

FIG. 4. As in Fig. 2 but for the case $\kappa_{d}>0\left(\varpi_{0}^{+}=1.15\right.$ and $\left.\varpi_{0}^{-}=-0.75\right)$.

The vortices and dipole velocities, $\mathbf{V}^{ \pm}(t)$ and $\mathbf{V}_{d}(t)$, respectively, can be computed from the vortices and dipole trajectories $\mathbf{R}^{ \pm}(t)$ and $\mathbf{R}_{d}(t)$,

$$
\mathbf{V}^{ \pm} \equiv \frac{d \mathbf{R}^{ \pm}}{d t} \quad \text { and } \quad \mathbf{V}_{d} \equiv \frac{d \mathbf{R}_{d}}{d t}
$$

This choice is, in practice, more precise than obtaining $\mathbf{V}_{d}$ from the velocity of every vortex defined as (1) but replacing $\mathbf{r}(\mathbf{x}, t)$ with the velocity of the fluid particles $\mathbf{u}(\mathbf{x}, t)$, because $\mathbf{V}^{ \pm}$is very small as compared with $\mathbf{u}$, approximately $O\left(\mathbf{V}^{ \pm}\right) / O(\mathbf{u}) \sim 10^{-3}$. 
The trajectory curvature $\kappa$ (e.g., Gill 1982, section 7.10) of the vortices or dipole is obtained from the Frenet-Serret formula:

$$
\kappa \equiv \mathbf{N} \cdot \frac{\partial \boldsymbol{S}}{\partial S}, \quad \mathbf{S} \equiv \frac{\boldsymbol{V}}{|\boldsymbol{V}|}, \quad \text { and } \quad \mathbf{N} \equiv \mathbf{k} \times \mathbf{S}
$$

where $\mathbf{S}$ and $\mathbf{N}$ are the unit vectors tangent and normal to the dipole trajectory, respectively.

\section{1) CASE $\kappa_{d}<0$}

The trajectories $\mathcal{R}^{ \pm}$are shown in Fig. 2. The negative dipole trajectory in this case, where the PV anomalies have the same magnitude $\left(\varpi_{0}^{+}=-\varpi_{0}^{-}\right)$, is due to the different vertical displacement of isopycnals $\mathcal{D}$ in each vortex, that is, to the different vertical size of the gyres. The south-north vertical cross section in Fig. 5a, displaying the contours of $\mathcal{D}$ and vertical vorticity $\zeta$, shows this vertical asymmetry. The isopycnals of the cyclone (right-hand side in Fig. 5a) are displaced vertically inward in the cyclone core, that is, $\operatorname{sgn}\{\mathcal{D}(x, y, z)\}=\operatorname{sgn}\{z\}$ (shrinking), whereas the isopycnals of the anticyclone (left-hand side) are displaced vertically outward in anticyclone core; that is, $\operatorname{sgn}\{\mathcal{D}(x, y, z)\}=\operatorname{sgn}\{z\}$ (stretching). Thus, the anticyclone has a vertical extent larger than the cyclone and, since the magnitude of $\varpi_{0}^{ \pm}$are identical for both vortices, it also has a larger relative vorticity (Fig. 6). As a consequence of this vertical extent and vorticity asymmetry, the anticyclone dominates the cyclone and moves along a trajectory with a negative curvature around a center common to both vortices.

The time evolution of the angle of $\mathbf{V}^{ \pm}=\left(U^{ \pm}, V^{ \pm}\right)$ with the $x$ axis $\Theta^{ \pm}(t) \equiv \arctan \left[V^{ \pm}(t) / U^{ \pm}(t)\right]$ is shown in Fig. 7a. A linear fit using a gradient-expansion least squares method gives $\bar{\Theta}(t) \equiv 1 / 2\left[\Theta^{+}(t)+\Theta^{-}(t)\right]=\bar{\Theta}_{0}+$ $\bar{\Omega} t$ (variance $\hat{\sigma}^{2}=5.72 \times 10^{-5}$ ) where the frequency $\bar{\Omega} \equiv d \bar{\Theta} / d t \simeq-3.36 \times 10^{-2}$ (i.p.) $)^{-1}$. Thus, the dipole moves clockwise tracing a circle with a time-averaged curvature $\overline{\kappa_{d}}=-0.161$, and a time-averaged period $\bar{T}=$ $2 \pi / \bar{\Omega} \simeq 186$ i.p.

The time evolution of $\mathbf{V}^{ \pm}$is shown in Fig. 8. The amplitude of $\mathbf{V}^{ \pm}$is several orders of magnitude smaller than the maximum fluid velocity (which is of order 1). Consistent with the dipole trajectory curvature the cyclone has larger speeds $\left\{\left|\boldsymbol{V}^{+}\right|(t) \subset[2.34,2.49] \times 10^{-3}\right\}$ than the anticyclone $\left\{\left|\boldsymbol{V}^{-}\right|(t) \subset[1.83,2.00] \times 10^{-3}\right\}$. The time-averaged speed of the dipole is $\mid \overline{\mathbf{V}_{d} \mid} \simeq$ $2.20 \times 10^{-3}$.

The deformation of the 3D dipole can be also inferred from Fig. 1. The surfaces of $\varpi$ at the lower layers of the vortices are displaced inward (toward the dipole axis) periodically so that the vortices acquire the shape

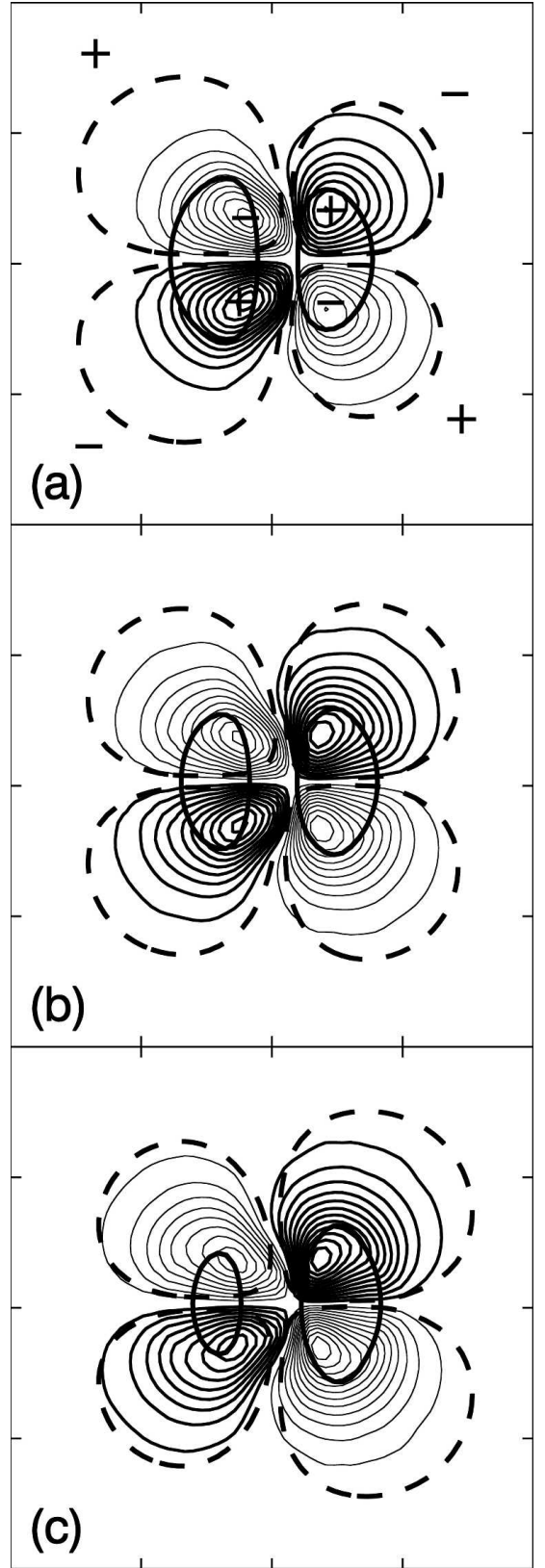

FIG. 5. South-north vertical cross section of $w$ at the $x$ index $i_{X}=12\left[x=2 \pi\left(i_{X}-1\right) / 64-\pi \simeq-2.0\right]$ for the cases (a) $\kappa_{d}<0$, (b) $\kappa_{d} \cong 0$, and (c) $\kappa_{d}<0$ at $t=6$ i.p. Axis domain $y \in[-\pi, \pi] c$, and $z \in[-\pi, \pi]$. Medium thick lines indicate upwelling $(w>0)$ and thin lines downwelling $(w<0)$ (minimum contour is $\pm 2.5 \times$ $10^{-5}$ and $\mathcal{D}= \pm 2.5 \times 10^{-5}$ ). The contours of $\mathcal{D}= \pm 1 \times 10^{-2}$ (dashed thick lines) and $\zeta= \pm 1 \times 10^{-2}$ (thickest lines) are included. Zero contour lines are always omitted.

of a vertical banana, the cyclone experiencing a larger deformation than the anticyclone. This is related to internal oscillations in the dipole (described below in section $3 \mathrm{~g}$ ). Such banana-shaped dipoles have been already observed in full 3D numerical simulations (Beck- 


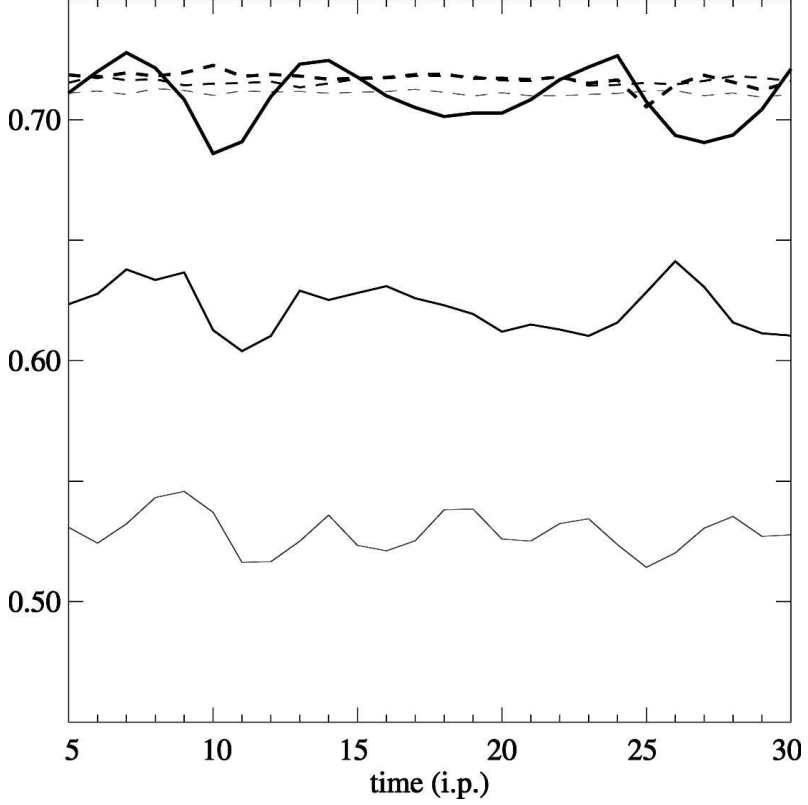

FIG. 6. Time series (from $t=5$ to 30 i.p.) of the maximum dimensionless relative vorticity $\zeta / f$ in the cyclone (continuous line) and anticyclone (dashed line) for the cases $\kappa_{d}<0$ (thin line), $\kappa_{d} \cong 0$ (medium thick line), and $\kappa_{d}>0$ (thickest line). The cyclone and the anticyclone are defined, here and in the following figures, as those locations having $\varpi_{0}^{ \pm}= \pm 5 \times 10^{-2}$.

ers et al. 2002) and also experimentally in a linearly stratified nonrotating fluid (Praud and Fincham 2005).

\section{2) $\mathrm{CASES} \kappa_{d} \cong 0$ AND $\kappa_{d}>0$}

The dipole, in the case $\kappa_{d} \cong 0$ and due to the increase of $\varpi_{0}^{+}$, has a quasi-straight trajectory (Fig. 3). The two vortices now have a similar isopycnal vertical displacement $|\mathcal{D}(x, y, z)|$ (Fig. 5b). However, the relative vorticity is still larger in the anticyclone than in the cyclone (Fig. 6). For this reason the trajectories $\mathbf{R}^{ \pm}$are not exactly straight (Figs. 3 and $7 \mathrm{~b}$ ) but have a small frequency $\bar{\Omega} \equiv-2.75 \times 10^{-3}$, obtained from the linear fit of $\bar{\Theta}(t) \equiv 1 / 2\left[\Theta^{+}(t)+\Theta^{-}(t)\right]=\bar{\Theta}_{0}+\bar{\Omega} t(\hat{\sigma}=2.70 \times$ $10^{-5}$, Fig. $\left.7 \mathrm{~b}\right)$, a very large period $\tilde{T} \simeq 2285$ i.p., and a small clockwise rotation $\left(\overline{\kappa_{d}} \equiv-0.014\right)$. The speed of displacement of the vortices $\left|\mathbf{V}^{ \pm}\right|$(Fig. 8) are similar $\left\{\left|\mathbf{V}^{+}\right|(t) \subset[2.31,2.48] \times 10^{-3}\right.$ and $\left|\mathbf{V}^{-}\right|(t) \subset[2.27,2.46]$ $\left.\times 10^{-3}\right\}$ because of the small dipole frequency $\bar{\Omega}$. The time-averaged speed of the dipole is somewhat larger than in the case $\kappa_{d}<0$, with $\overline{\left|\mathbf{V}_{d}\right|} \simeq 2.40 \times 10^{-3}$.

Increasing, again, $\varpi_{0}^{+}$by an amount $\Delta \varpi_{0}^{+}=0.2$, now the cyclone dominates because of its larger vertical extent with respect to the anticyclone (Fig. 5c). The relative vorticity $\zeta$ is approximately equal in both vortices (Fig. 6). The dipole acquires a clockwise rotation (Fig. 4) with $\overline{\kappa_{d}} \equiv+0.097$, a positive frequency $\bar{\Omega} \equiv+2.57 \times$

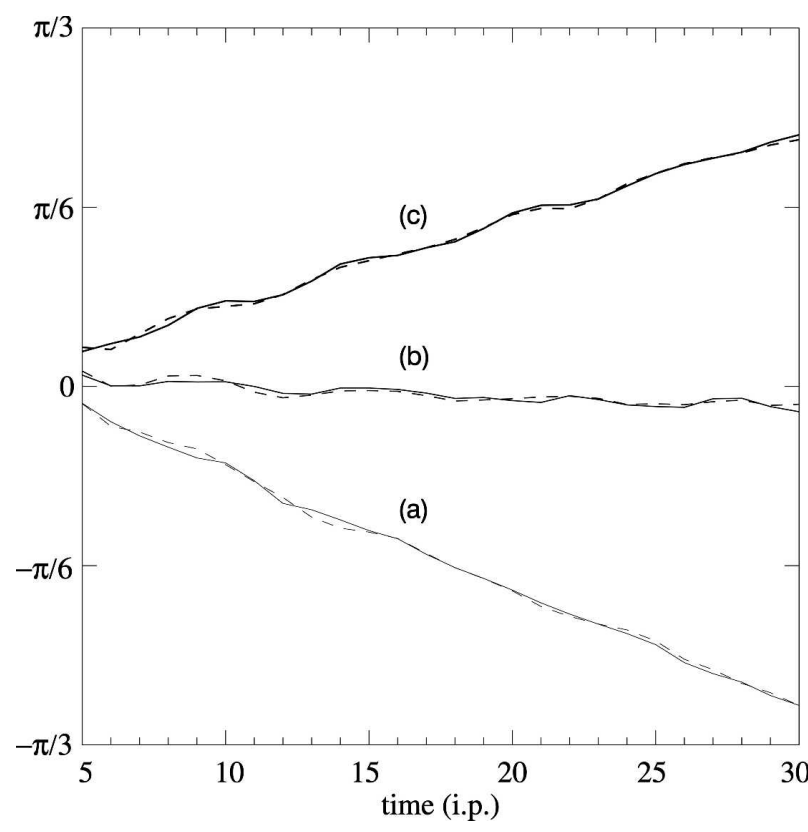

FIG. 7. Time series (from $t=5$ to 30 i.p.) of the angle of $\mathbf{V}^{ \pm}$with the $x$ axis for the cyclone (continuous line) and the anticyclone (dashed line) for the cases (a) $\kappa_{d}<0$, (b) $\kappa_{d} \cong 0$, and (c) $\kappa_{d}>0$.

$10^{-2}\left(\hat{\sigma}^{2}=3.49 \times 10^{-5}\right.$, Fig. $\left.7 \mathrm{c}\right)$, and a period $\bar{T} \simeq 244$ i.p. Consistent with the positive curvature the anticyclone speed $\left|\mathbf{V}^{-}\right|(t) \subset[2.65,2.87] \times 10^{-3}$ is larger than $\left|\mathbf{V}^{+}\right|(t) \subset[2.31,2.46] \times 10^{-3}$ (Fig. 8). In this case the dipole has the largest time averaged speed of the three cases, $\overline{\left|\mathbf{V}_{d}\right|} \simeq 2.60 \times 10^{-3}$.

\section{c. Horizontal velocity}

\section{1) CASE $\kappa_{d}<0$}

The magnitude of the total horizontal velocity $\mathbf{u}_{h}$ (Fig. 9) reaches maximum values of 1.60 at the middle layer $(z=0)$ and along the dipole axis. These maxima are associated with the confluence and difluence of the horizontal flow in the dipole entrance (rear part) and exit region (front part), respectively. The minima of $\left|\mathbf{u}_{h}\right|$ are located in the vortex centers where the small fluid speed is similar to the dipole displacement speed.

\section{2) $\operatorname{CASES} \kappa_{d} \cong 0$ AND $\kappa_{d}>0$}

The maximum values of $\left|\mathbf{u}_{h}\right|$ are 1.73 (case $\kappa_{d} \cong 0$, Fig. 10) and 1.85 (case $\kappa_{d}>0$, Fig. 11). The increase of $\left|\mathbf{u}_{h}\right|$ in the dipole axis relative to the reference case is due to the larger PV gradients, which increase the confluence and difluence of the horizontal flow.

The effect of the mirror dipole (due to the triply periodic boundary conditions) is very minor. This can be deduced, for example, from Fig. 9 where only the 


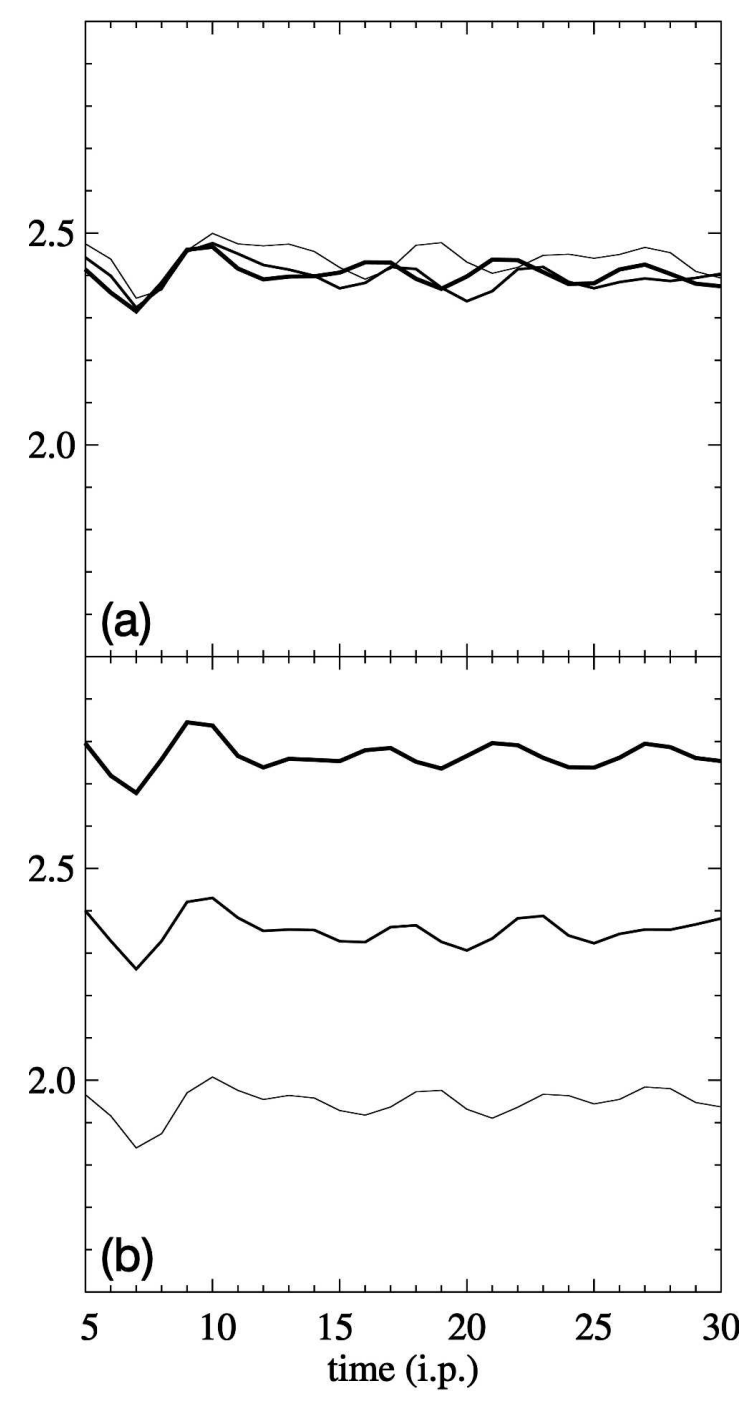

FIG. 8. Time series (from $t=5$ to 30 i.p.) of (a) $\mathbf{V}^{+}$and (b) $\mathbf{V}^{-}$ for the cases $\kappa_{d}<0$ (thin line), $\kappa \cong 0$ (medium thick line), and $\kappa_{d}>0$ (thickest line). The vertical axis is in units of $10^{-3}$.

smallest contour of $\left|\mathbf{u}_{h}\right|$ in the dipole is connected with the one in the mirror dipole. The triply periodic domain is large enough to largely avoid the influence of neighboring dipoles.

\section{d. Particle trajectories}

Introducing the streamfunction $\boldsymbol{\Phi}$ for the horizontal and area-preserving flow at $z=0$,

$$
\mathbf{u}_{h}=-\mathbf{k} \times \nabla_{h} \mathbf{\Phi},
$$

the co-moving streamfunction $\boldsymbol{\Psi}$, in a reference frame connected to the dipole, may be written as

$$
\boldsymbol{\Psi}=\boldsymbol{\Phi}-\left(\mathbf{r}_{h} \times \mathbf{V}_{d}\right) \cdot \mathbf{k},
$$

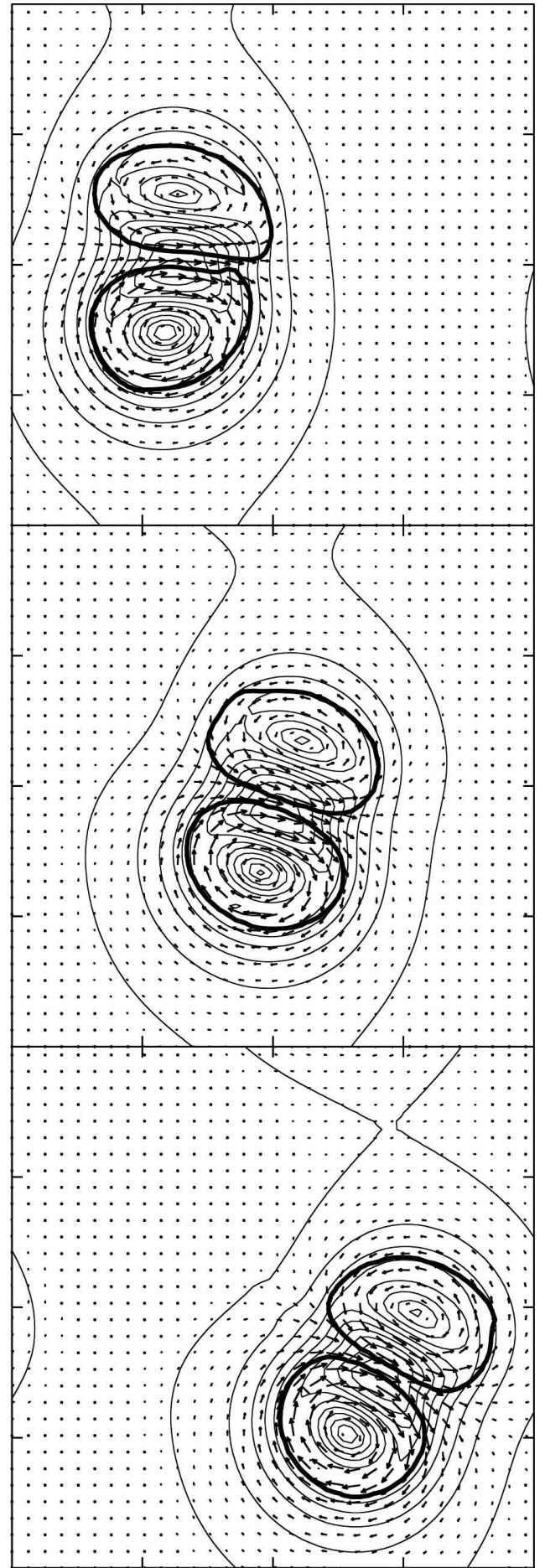

FIG. 9. Time evolution ( $t=6,12$, and 18 i.p.) of the horizontal distribution of $\mathbf{u}_{h}$ at the middle layer $z=0$ for the case $\kappa_{d}<0$. Horizontal domain is $x, y \in[-\pi, \pi] c$. Only every other vector has been plotted. The contour $\varpi_{0}= \pm 5 \times 10^{-2}$ at $z=0$ (thick lines) is included for reference. Thin lines indicate the speed $\left|\mathbf{u}_{h}\right|(\Delta=$ $1.5 \times 10^{-1}$, with a minimum contour of $1 \times 10^{-1}$ ). 


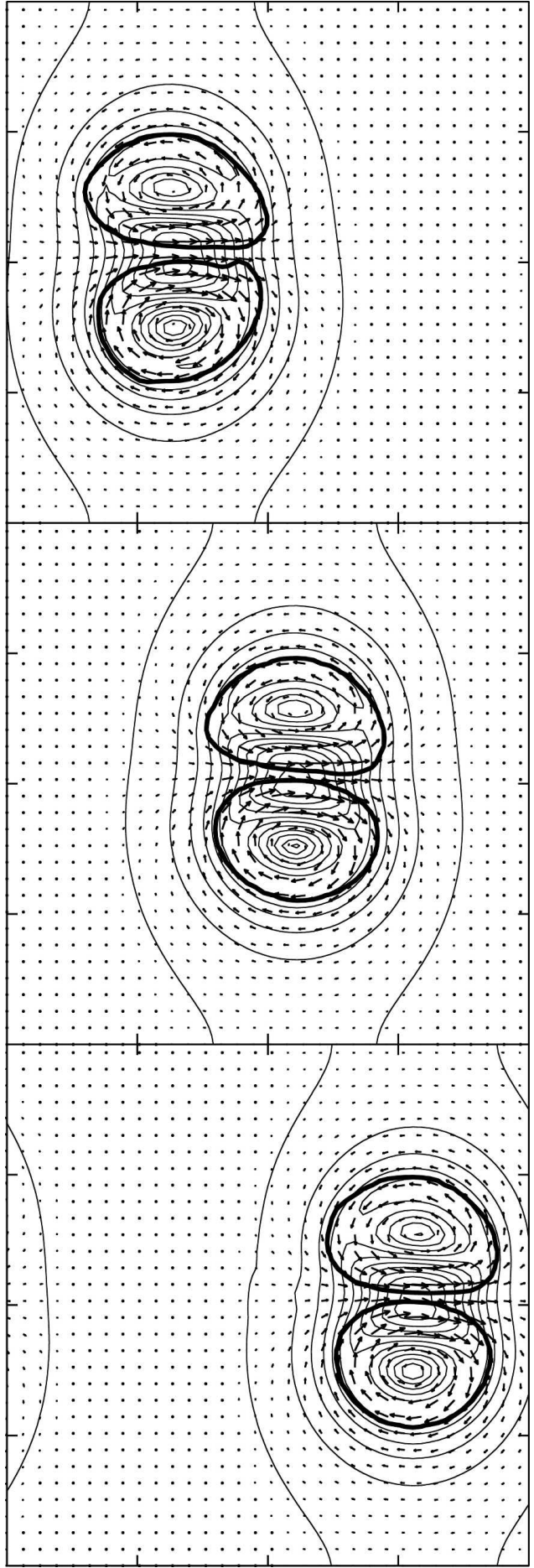

FIG. 10. As in Fig. 9 but for the case $\kappa_{d} \cong 0$.

where $\mathbf{V}_{d}=\left(u_{d}, v_{d}\right)$ is the dipole velocity and $\mathbf{r}_{h}=(x$, $y)$ is the position vector. The distribution of $\boldsymbol{\Psi}(x, y)$ (Fig. 12) shows the elliptical shape of the separatrix of the dipole. The separatrix was fitted to the zero contour of $\boldsymbol{\Psi}$ through the minimization of

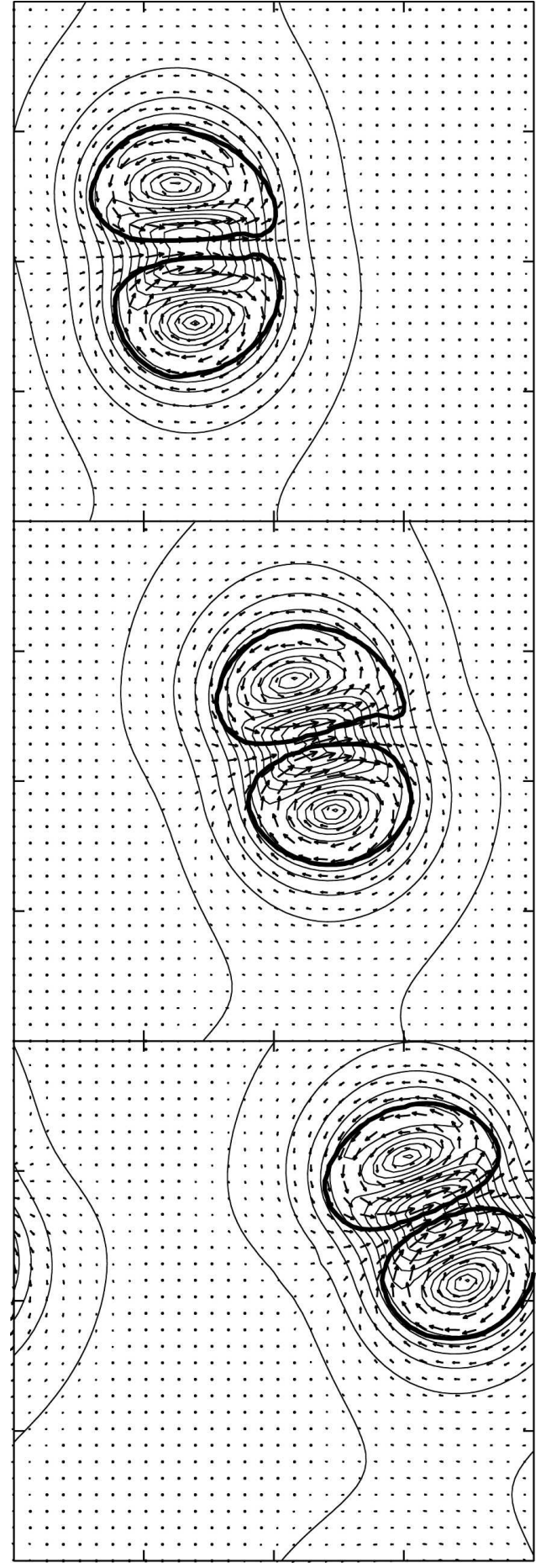

FIG. 11. As in Fig. 9 but for the case $\kappa_{d}>0$.

$$
\sum_{i=1}^{N_{p}} \sum_{j=1}^{N_{p}}\left[\Psi_{e}(i, j)-\overline{\Psi_{e}}\right]^{2}
$$

where $\Psi_{e}(i, j)$ are the values of $\Psi$ in the $N_{p}$ points of the separatrix. The ellipse aspect ratio was matched to 


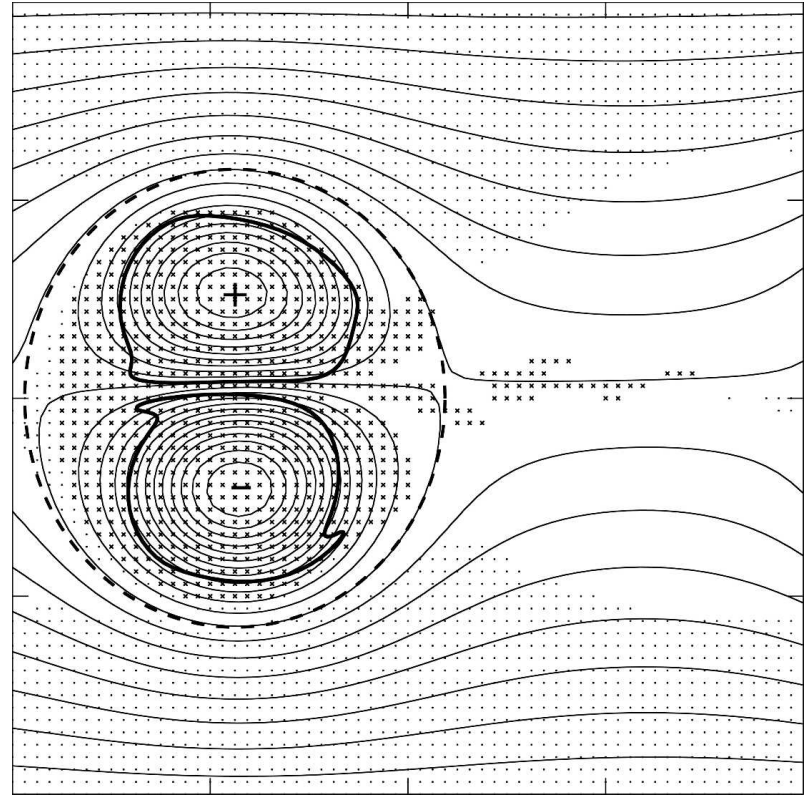

FIG. 12. The co-moving streamfunction $\boldsymbol{\Psi}$ on the plane $z=0$ for the case $\kappa=0$ (thin lines; $\Delta= \pm 1.0 \times 10^{-5}$; the zero contour is included). The horizontal domain is $x, y \in[-\pi, \pi] c$. The separatrix of the dipole (dashed line), and the outermost positive and negative PV contours are included (thick lines).

$R_{y} / R_{x} \simeq 1.09$, where $R_{y}$ and $R_{x}$ are the ellipse radii along the $x$ and $y$ axes. Elliptical separatrices in barotropic dipoles have been found both numerically (Hesthaven et al. 1995) and theoretically (Khvoles et al. 2005).

Dipoles are almost impermeable to outward particle fluxes. The separatrix may be regarded as a vortex frontier (closed streamline) between the interior and exterior flow, the latter resembling the 2D irrotational flow around an elliptical cylinder. Three transport regimes, depending on the length $L_{0}$ and geometry of the path of the particles, can be inferred from Figs. 12 and 13, namely (i) particles with $\pi / 2 \leq L_{0}<\pi$ (dots in Fig. 12) and nonvortical path (Fig. 13a), (ii) particles with $L_{0}<$ $\pi / 2$ (blanked region in Fig. 12) and vortical path (Fig. $13 \mathrm{~b}$ ), and (iii) particles with $L_{0} \geq \pi$ ( $\times$ symbols in Fig. 12) and vortical path (Fig. 13c). The particles located inside the separatrix experience the largest trajectories. A few particles initially located outside the separatrix can be trapped inside the dipole for long times and transported by the vortex motion over large distances $L_{0} \geq \pi$ (Fig. 12). Some permeability and particle advection can be inferred from the motion of the filaments that are released during the first stages of the dipole evolution. These filaments are too thin to modify the PV field and can be considered as passive Lagrangian tracers located outside the vortical flow. Some of them are left behind but most of them move with the dipole (Figs. 2, 3, and 4 at $t=18$ i.p.).

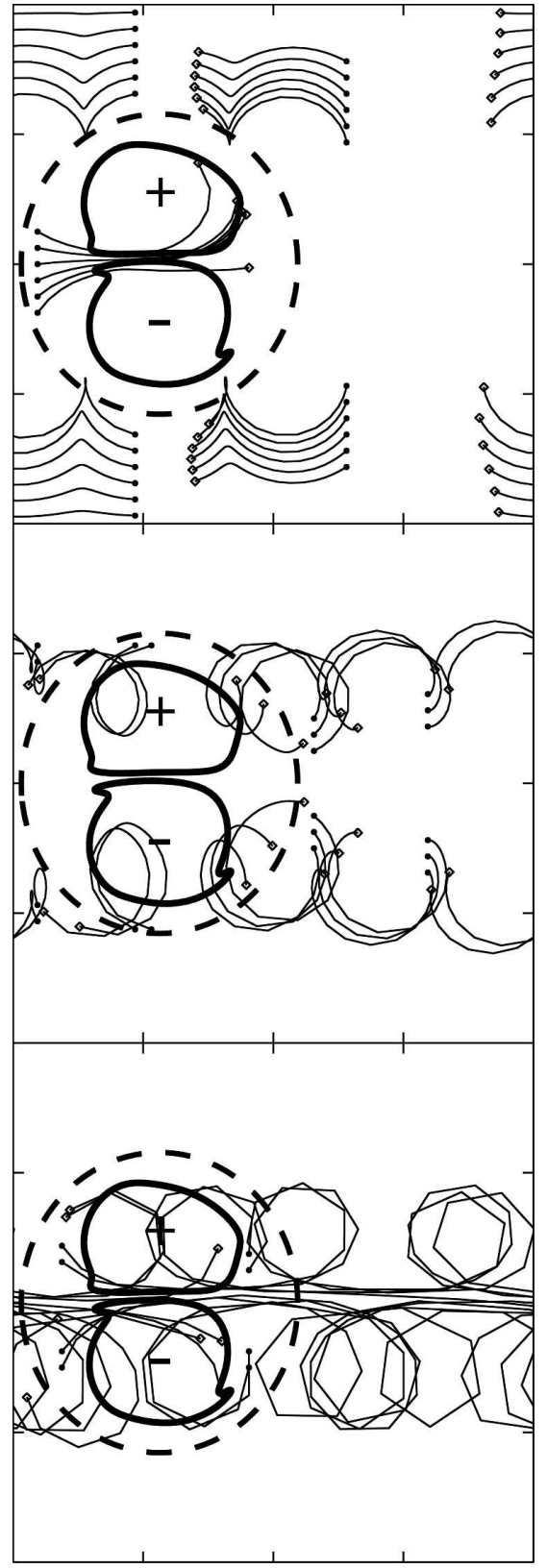

FIG. 13. Particle trajectories: particles having moved a distance (a) $\pi / 2 \leq L_{0}<\pi$ with a nonvortical path, (b) $L_{0}<\pi / 2$ with a vortical path, and (c) $L_{0} \geq \pi$ with a vortical path. Solid dots and diamonds indicate the initial and final positions of the particle, respectively. The separatrix of the dipole (dashed line) is included.

\section{e. Vertical velocity}

\section{1) CASE $\kappa_{d}<0$}

The vertical velocity $w$ has an octupolar structure in the 3D space, or quadrupolar pattern in the horizontal or vertical 2D planes, of alternating upwelling and downwelling cells (Fig. 14). The horizontal distribution 


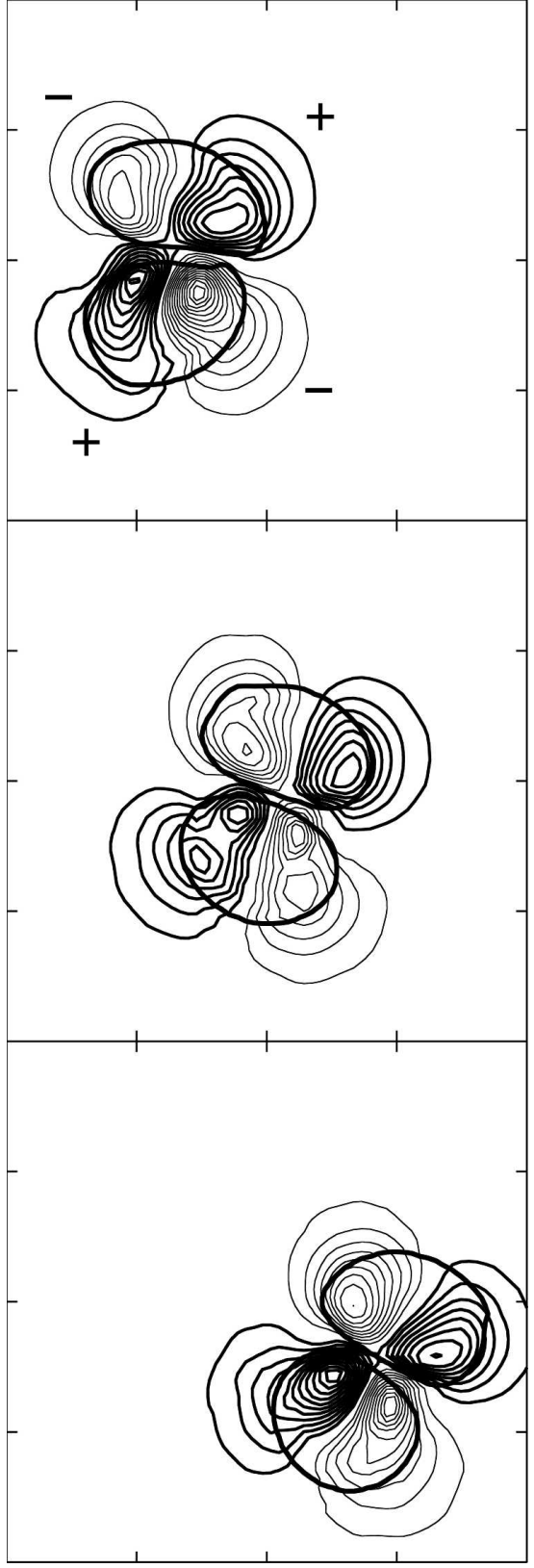

FIG. 14. Time evolution ( $t=6,12$, and 18 i.p.) of the horizontal distribution of $w$ in the grid plane $i_{Z}=27\left[z=2 \pi\left(i_{Z}-1\right) / 64-\right.$ $\pi \simeq-0.59$ ] for the case $\kappa_{d}<0(w>0$, medium thick lines; $w<$ 0 , thin lines; $\Delta= \pm 2.5 \times 10^{-5}$; minimum contour of $\pm 2.5 \times 10^{-5}$ ). Horizontal domain is $x, y \in[-\pi, \pi] c$. The contour $\varpi_{0}= \pm 5 \times$ $10^{-2}$ at $z=0$ (thickest lines) is included.

of $w$ in an isolated mesoscale ellipsoidal vortex has a quadrupolar pattern (VD03) However, when two ellipsoidal vortices get close enough to form a vortex dipole, they lose their PV symmetry along the major axis: the PV contours of the outermost part of every vortex acquire a larger curvature than the PV contours located close to the dipole axis. Thus, the vortices no longer

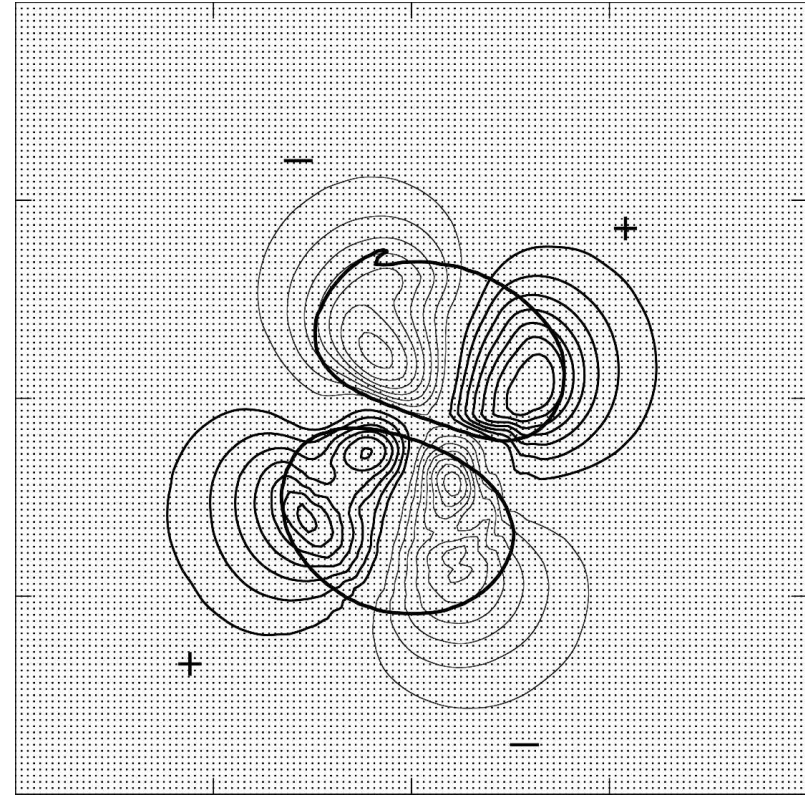

Fig. 15. Horizontal distribution of $w$ at $t=12$ i.p. on the plane $i_{Z}=53\left[z=2 \pi\left(i_{Z}-1\right) / 128-\pi \simeq-0.59\right]$ for the case $\kappa_{d}<0$ $\left(w>0\right.$, medium thick lines; $w<0$, thin lines; $\Delta= \pm 2.5 \times 10^{-5}$; the minimum contour is $\left.\pm 2.5 \times 10^{-5}\right)$. The horizontal domain is $x, y \in[-\pi, \pi] c$. The contour $\varpi_{0}= \pm 5 \times 10^{-2}$ at $z=0$ (thickest lines) is included. The dots indicate the grid points.

remain ellipsoidal and their individual quadrupolar $w$ pattern is lost.

Instead, the largest PV contour curvature occurs at both sides of the dipole axis, at the rear and front parts, and hence the new quadrupolar $w$ pattern.

The horizontal distribution of $w$, in a similar simulation but with higher numerical resolution $\left(128^{3}\right.$ grid points, Fig. 15), shows that the quadrupolar structure remains similar to the lower resolution case (Fig. 14; $t=$ 12 i.p.). A small perturbation appears now in the negative cell of the anticyclone. Results using higher numerical resolution $\left(256^{3}\right.$ grid points $)$ suggest that this perturbation could be related to the spontaneous emission of inertia-gravity wave packets by the balanced dipole flow.

Extreme values of $w$ in the dipole reaches $\max \{w\}=$ $\{25.0,17.5,27.5\} \times 10^{-5}$ and $\min \{w\}=-\{32.5,17.5$, $25.0\} \times 10^{-5}$, at times $t=\{6,12,18\}$ i.p., respectively (Fig. 14). The time series of $\max \{|w|\}$ (Fig. 16) display a number of time oscillations. It is shown below that these are a consequence of the internal dipole motion, namely the small fluctuations in the vortex trajectories seen in Fig. 1. As a consequence $w$ in the vortices experience important changes both in pattern and magnitude. For example, both single extrema of $w$ at $t=6$ i.p. are split into two extrema at $t=12$ i.p. (Fig. 14), and 


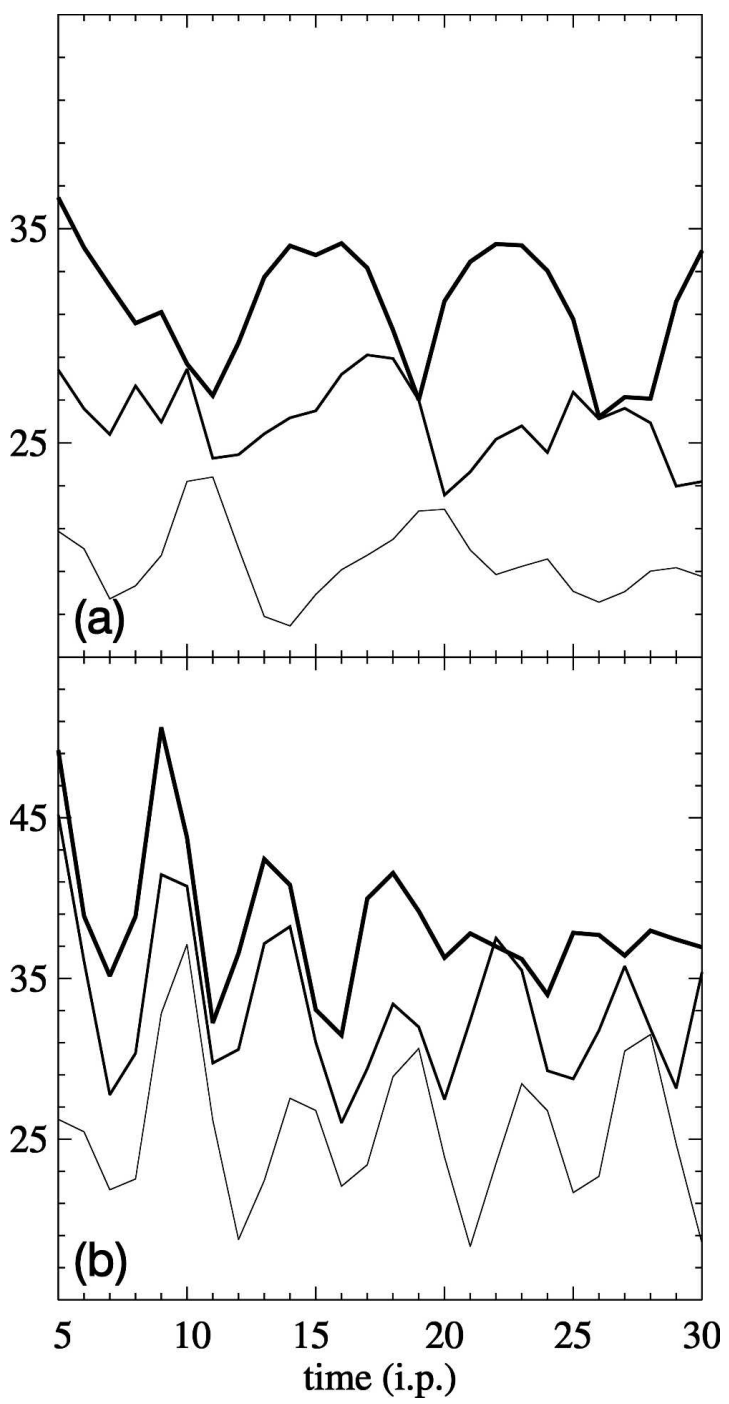

FIG. 16. Time series of the extrema of $|w|$ in the (a) cyclone and (b) anticyclone for the three cases $\kappa_{d}>0$ (thin lines), $\kappa \cong 0$ (medium thick line), and $\kappa_{d}<0$ (thickest line). The vertical axis is in units of $10^{-5}$.

the magnitudes of the maxima decrease approximately $40 \%$ (Fig. 16).

The vertical cross section (Fig. 5a) shows the vertical component of the octupolar structure of $w$. As is typical of mesoscale geophysical flows the motion is largely horizontal, with a ratio $O\left(\left|\mathbf{u}_{h}\right|\right) / O(w) \sim 10^{4}$. The anticyclone has larger vertical gradients of $w$ than the cyclone, consistently with having larger $|w|$, as is clearly seen in the time series of $\max \{|w|\}$ (Fig. 16).

\section{2) CASES $\kappa_{d} \cong 0$ AND $\kappa_{d}>0$}

In these cases $w$ also displays an octupolar pattern (Figs. 17, 5b, 18, and 5c). The distributions of $w$ are very

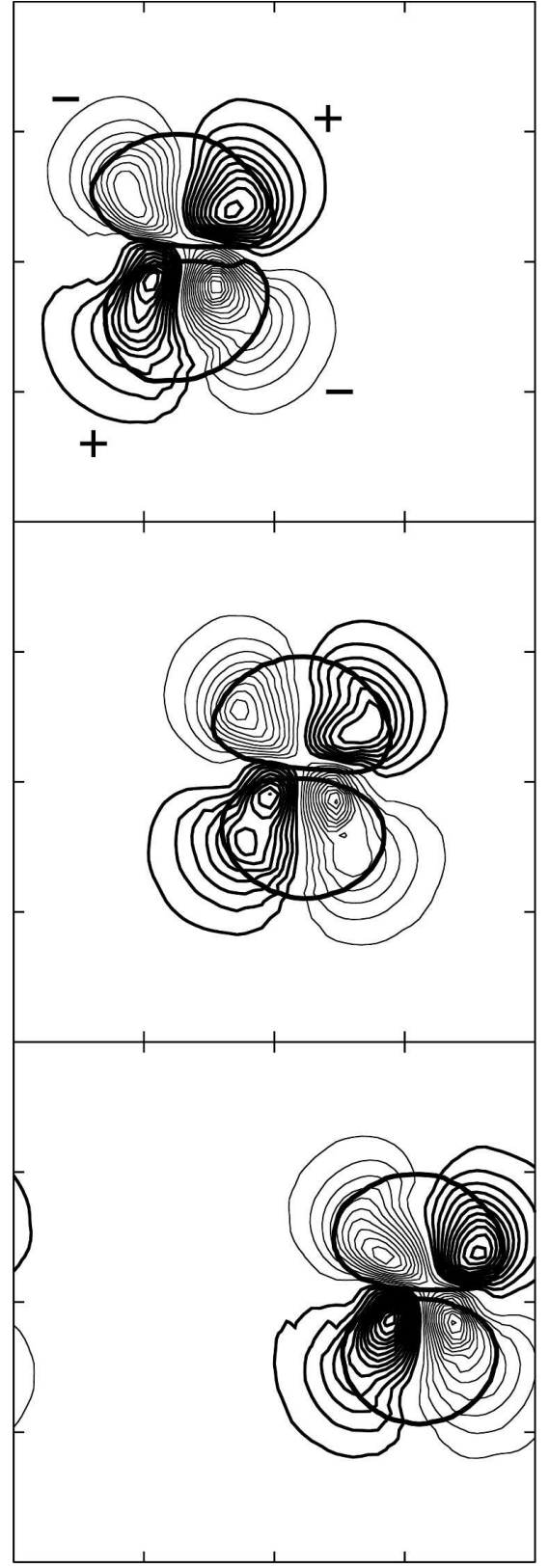

FIG. 17. As in Fig. 14 but for the case $\kappa_{d} \cong 0$.

similar to those of the reference simulation and only changes in magnitude are observed. The $w$ extrema in the vortices are $\max \{w\}=\{25.0,20.0,32.5\} \times 10^{-5}$ and $\min \{w\}=-\{35.0,30.0,32.5\} \times 10^{-5}\left(\right.$ case $\kappa_{d} \cong 0$, Fig. 17), and $\max \{w\}=\{32.5,27.5,37.5\} \times 10^{-5}$ and $\min \{w\}=-\{37.5,32.5,37.5\} \times 10^{-5}\left(\right.$ case $\kappa_{d}>0$, Fig. $18)$, at times $t=\{6,12,18\}$ i.p., respectively. As expected, the larger $\varpi_{0}^{+}$in the cyclone, i.e., larger $\mathcal{R}^{+}$(Fig. 6 ), increases $\partial w / \partial z$, and the anticyclone has the largest values of $w$ as observed in the time series of $\max \{|w|\}$ (Fig. 16). 


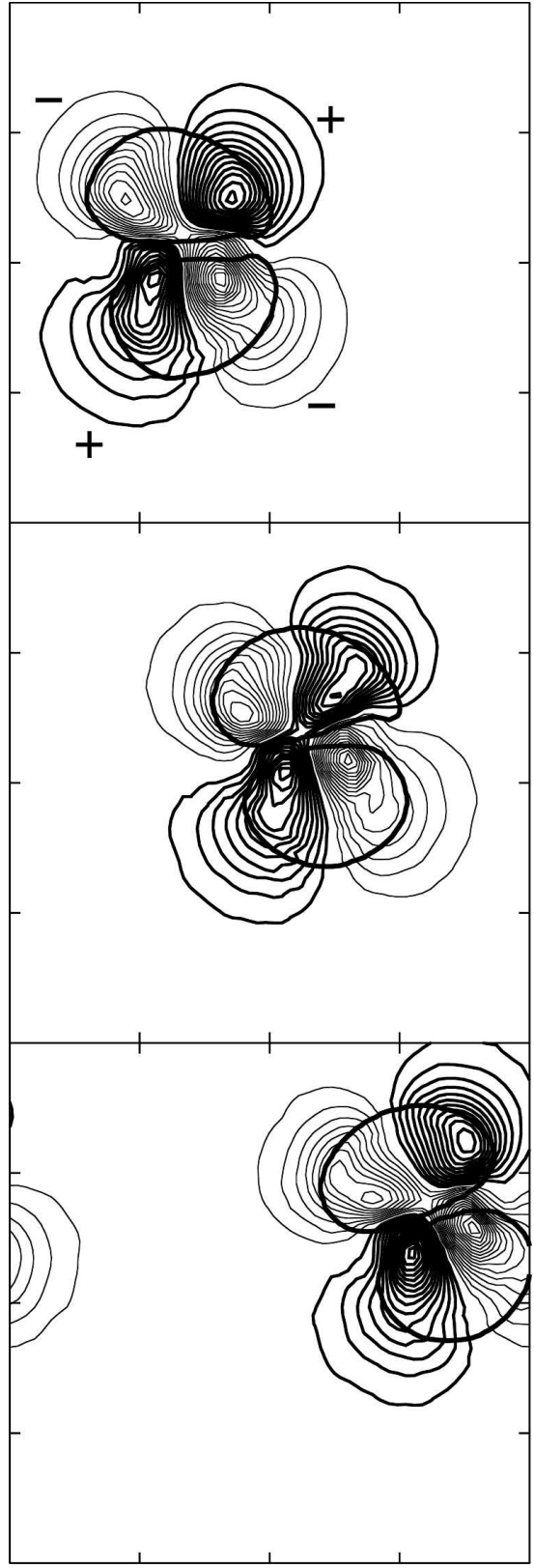

FIG. 18. As in Fig. 14 but for the case $\kappa_{d}>0$.

\section{f. Vertical velocity and vertical vorticity}

The quadrupolar distribution of $w$ can be diagnosed from the main forcing term in the generalized omega equation, which for mesoscale geophysical flows can be approximately interpreted (Pallàs-Sanz and Viúdez 2005 ) as proportional to the horizontal advection by the vertical shear velocity of the vertical vorticity:

$$
\begin{aligned}
& \left(f \zeta_{\mathrm{ph}}+N^{2} \nabla_{h} \mathcal{D}\right) \cdot \nabla_{h}^{2} \mathbf{u}_{h}=\left(2 f \zeta_{\mathrm{ph}}-f \zeta_{\mathrm{ph}}^{\prime}\right) \cdot \nabla_{h}^{2} \mathbf{u}_{h} \\
\simeq & 2 f\left(\mathbf{k} \times \mathbf{u}_{h z}\right) \cdot\left(\mathbf{k} \times \nabla_{h} \zeta\right)=2 f \mathbf{u}_{h z} \cdot \nabla_{h} \zeta,
\end{aligned}
$$

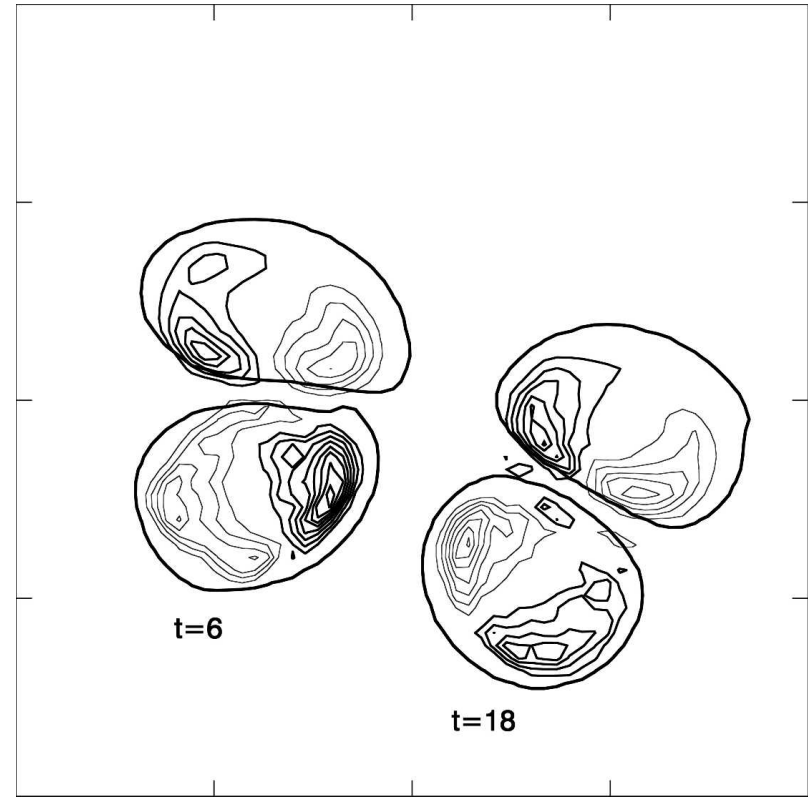

FIG. 19. Horizontal distribution of $\mathbf{u}_{h z} \cdot \boldsymbol{\nabla}_{h} \zeta$ (thick/thin lines mean positive/negative values) at $z=-0.59$ and at times $t=6$ and 18 i.p. for the case $\kappa_{d}<0\left(\Delta=0.5 \times 10^{-4}\right.$ and minimum contour of $\left.0.5 \times 10^{-4}\right)$. Horizontal domain is $x, y \in[-\pi, \pi] c$. The contour of $\varpi_{0}= \pm 5 \times 10^{-2}$ at $z=0$ (thickest line) is included for reference.

where $N^{2} \nabla_{h} \mathcal{D}=f \mathcal{\zeta}_{\text {ph }}^{g}$, and $\zeta_{\text {ph }} \equiv-v_{z} \mathbf{i}+u_{z} \mathbf{j}$ is the horizontal pseudovorticity. The horizontal distribution of $\mathbf{u}_{h z} \cdot \boldsymbol{\nabla}_{h} \zeta$ is shown in Fig. 19. In the lower layers $(z<0)$ the velocity shear $\mathbf{u}_{h z}$ is in the direction of $\mathbf{u}$. As the fluid particles close to the dipole axis move forward reaching the front, their $|\zeta|$ decreases because the decrease in shear vorticity is larger than the increase of curvature vorticity. Particles in the right (left) side of the dipole axis lose negative (positive) vorticity. Thus, $\mathbf{u}_{h z} \cdot \boldsymbol{\nabla}_{h} \zeta>0\left(\mathbf{u}_{h z} \cdot \nabla_{h} \zeta<0\right)$ in the right (left) side, which implies $w<0(w>0)$. A parallel argument applies to the rear part of the dipole. In the upper layers $(z>0)$ the velocity shear $\mathbf{u}_{h z}$ is in the direction of $-\mathbf{u}$, and therefore $\operatorname{sgn}\{w(z>0)\}=-\operatorname{sgn}\{w(z<0)\}$. The interpretation of $w$ in terms of $\mathbf{u}_{h z} \cdot \nabla_{h} \zeta$ predicts the correct sign of $w$ in both upper and lower layers, independent of whether the vertical shear $\mathbf{u}_{h z}$ is in the same or opposite direction to $\mathbf{u}_{h}$. The visual quasigeostrophic interpretation of $w$ in terms of $\mathbf{u}_{h} \cdot \nabla_{h} \zeta$ would however infer the wrong sign in the upper half of subsurface vortices or meanders where inverse baroclinicity is important.

\section{g. Horizontal ageostrophic velocity}

Using the inviscid horizontal momentum equation

$$
\frac{d \mathbf{u}_{h}}{d t}+f \mathbf{k} \times \mathbf{u}_{h}^{\prime}=0,
$$


the horizontal ageostrophic velocity $\mathbf{u}_{h}^{\prime} \equiv \mathbf{u}_{h}-\mathbf{u}^{g}$ can be expressed as

$$
\mathbf{u}_{h}^{\prime}=\frac{1}{f} \mathbf{k} \times \frac{d \mathbf{u}_{h}}{d t}=\frac{1}{f} \mathbf{k} \times\left(\frac{\partial \mathbf{u}_{h}}{\partial t}+\mathbf{u}_{h} \cdot \nabla_{h} \mathbf{u}_{h}+w \frac{\partial \mathbf{u}_{h}}{\partial z}\right),
$$

in terms of the local and the horizontal and vertical advective accelerations.

In the reference frame defined by the horizontal unit vectors tangent and normal to the flow, $\mathbf{s} \equiv \mathbf{u}_{h} / u$ and $\mathbf{n} \equiv \mathbf{k} \times \mathbf{s}\left(u \equiv\left|\mathbf{u}_{h}\right| \neq 0\right)$, the total horizontal and geostrophic velocities are $\mathbf{u}_{h}=u \mathbf{s}$, and $\mathbf{u}^{g}=u_{s}^{g} \mathbf{s}+u_{n}^{g} \mathbf{n}$, respectively. We define that the flow $\mathbf{u}_{h}$ is supergeostrophic (subgeostrophic) if $u>u_{s}^{g},\left(u<u_{s}^{g}\right)$. In the $(\mathbf{s}, \mathbf{n})$ reference frame the $\mathbf{n}$ component of the horizontal momentum balance (6) for steady flow (gradient balance) is

$$
u^{2} \kappa=-f\left(u-u_{s}^{g}\right),
$$

where $\kappa \equiv \mathbf{k} \cdot \nabla_{h} \times \mathbf{s}=\mathbf{n} \cdot\left(\mathbf{s} \cdot \nabla_{h} \mathbf{s}\right)$ is the streamline curvature. Hence, positive (negative) streamline curvature implies subgeostrophic (supergeostrophic) flow.

\section{1) CASE $\kappa_{d}<0$}

The horizontal ageostrophic velocity at the depth of maximum speed $(z=0)$ is shown in Fig. 20. In general $\mathbf{u}_{h}^{\prime}$ is opposite to $\mathbf{u}_{h}$ in the cyclone, but both point in the same direction in the anticyclone. Thus, consistent with (8), the cyclone is subgeostrophic and the anticyclone is supergeostrophic. The largest $\left|\mathbf{u}_{h}^{\prime}\right|$ occur in the anticyclone (reaching up to 0.35 , two times larger than in the cyclone), consistent with the spatial average time series of $\mathcal{R}^{ \pm}$(Fig. 6) and the time series of the extrema of $\left|\mathbf{u}_{h}^{\prime}\right|$ (Fig. 21).

The ageostrophic characteristics of the dipole are preserved during its time evolution, indicating the soundness of the gradient balance (8) when the local acceleration [first term in the rhs of (7)] is small compared to the horizontal advective acceleration [second term in the rhs of (7)]. The ratio between the horizontal advective and local accelerations in a simple kinematical model of a vortex whose center is rotating as $\mathbf{P}(t) \equiv$ $\mathbf{P}_{0} e^{i \Omega t}$, evaluated at a distance $\chi \mathbf{P}(t)$ (see the appendix), is

$$
\frac{\left|\mathbf{u} \cdot \nabla_{h} \mathbf{u}\right|}{|\partial \mathbf{u} / \partial t|}(\chi)=\frac{|\omega|}{|\Omega|} \frac{|\Omega-\omega(1-\chi)|}{|\Omega-\omega|},
$$

where $\omega$ is the frequency of the rotating fluid particles in the vortex, and $\Omega$ is the frequency of the vortex relative to its center of curvature. In our cases this ratio is large since $(1-\chi)|\omega| /|\Omega|=\left[1-\left(R_{d}-a_{m}\right) / R_{d}\right]|\omega| /|\Omega|=$

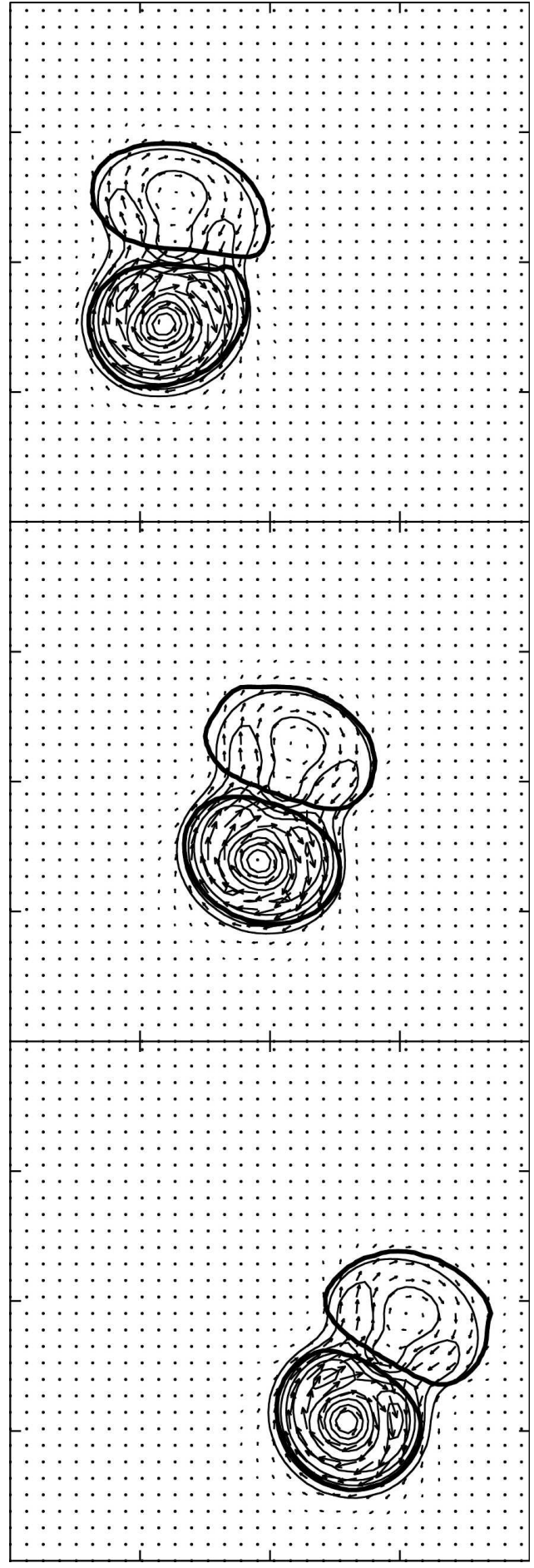

FIG. 20. Time evolution ( $t=6,12$, and 18 i.p.) of the horizontal distribution of $\mathbf{u}_{h}^{\prime}$ at $z=0$ for the case $\kappa_{d}<0$ (speed $\left|\mathbf{u}_{h}^{\prime}\right|$, thin line with $\Delta=0.5 \times 10^{-1}$, and minimum contour of $\left.10^{-1}\right)$. Horizontal domain is $x, y \in[-\pi, \pi] c$. Only every other vector has been plotted. The contour of $\varpi_{0}= \pm 5 \times 10^{-2}$ at $z=0$ (thick line) is included. 


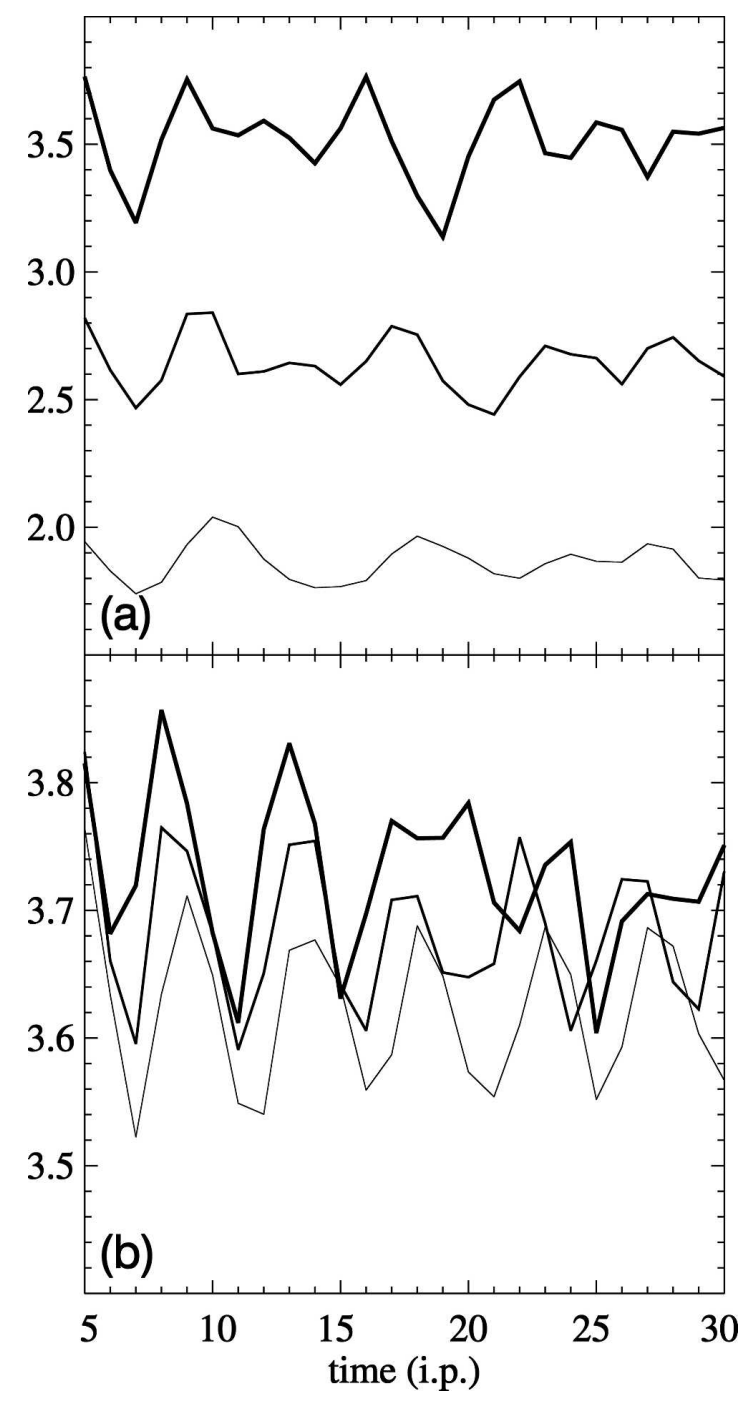

FIG. 21. Time series of the extrema of $\left|\mathbf{u}_{h}^{\prime}\right|$, in the (a) cyclone and (b) anticyclone for the cases $\kappa_{d}<0$ (thin line), $\kappa_{d} \cong 0$ (medium thick line), and $\kappa_{d}>0$ (thickest line). The vertical axis is in units of $10^{-1}$.

$\left(a_{m} / R_{d}\right)|\omega| /|\Omega| \sim 10^{-1}|\omega| /|\Omega| \gg 1$. Hence, the contribution to $\mathbf{u}_{h}^{\prime}$ due to the local acceleration is only a small fraction of the component due to the horizontal advective acceleration. On the other hand, the ratio between the horizontal advective acceleration and vertical advective acceleration [third term in the rhs of (7)],

$$
\frac{\left|\mathbf{u}_{h} \cdot \nabla_{h} \mathbf{u}_{h}\right|}{\left|w \partial \mathbf{u}_{h} / \partial z\right|}
$$

has an order of magnitude of $O\left(10^{4}\right)$. Thus, the most important contribution to $\mathbf{u}_{h}^{\prime}$ is due to the horizontal advective acceleration.

In the $(\mathbf{s}, \mathbf{n})$ reference frame, the horizontal advective acceleration may be decomposed into speed acceleration and centripetal acceleration:

$$
\mathbf{u}_{h} \cdot \nabla_{h} \mathbf{u}_{h}=\frac{\delta}{\delta s}\left(\frac{u^{2}}{2}\right) \mathbf{s}+u^{2} \kappa \mathbf{n},
$$

where $\delta / \delta s \equiv \mathbf{s} \cdot \nabla_{h}$ is the directional derivative along $\mathbf{s}$. The horizontal advective acceleration in the dipole is mainly due to the centripetal acceleration because it has a large $\mathbf{n}$ component (Fig. 22).

The distribution of $\mathbf{u}_{h}^{\prime}$ (Fig. 20) agrees with the pattern expected from the horizontal advective acceleration (Fig. 22). On one side, the advective acceleration in the vortices points to the center of the vortices since the centripetal acceleration is important. On the other side, along the dipole axis the speed acceleration is more important, which produces northward (southward) cross-stream ageostrophic flow entering (leaving) the speed maxima in the dipole axis. The maximum values of $u_{h}^{\prime} \equiv\left|\mathbf{u}_{h}^{\prime}\right|$ occur at both sides of the rear and front parts of the dipole axis, where both speed and centripetal accelerations contribute. The flow is more geostrophic at the dipole center where the total speed is maximum (seen as a minimum in $u_{h}^{\prime}$ in Fig. 20) because of the lack of curvature and speed acceleration (Fig. 22).

Introducing the unit tangent vector of the horizontal ageostrophic flow $\mathbf{s}^{\prime} \equiv \mathbf{u}_{h}^{\prime} / u_{h}^{\prime},\left(u_{h}^{\prime} \neq 0\right)$, the divergence of the ageostrophic flow $\boldsymbol{\nabla}_{h} \cdot \mathbf{u}_{h}^{\prime}$ can be decomposed into two contributions:

$$
\boldsymbol{\nabla}_{h} \cdot \mathbf{u}_{h}^{\prime}=-\partial w / \partial z=\mathbf{s}^{\prime} \cdot \nabla_{h} u_{h}^{\prime}+u_{h}^{\prime} \nabla_{h} \cdot \mathbf{s}^{\prime},
$$

where $\mathbf{s}^{\prime} \cdot \nabla_{h} u_{h}^{\prime}$ and $u_{h}^{\prime} \nabla_{h} \cdot \mathbf{s}^{\prime}$ are the speed acceleration and the confluence of the ageostrophic flow, respectively. Since the magnitude of the mesoscale vertical velocity is small, the ageostrophic speed acceleration and confluence have similar amplitudes but opposite signs (Figs. 23a,b), reaching extrema of $\{-5.9,6.1\}$ and $\{-6.6,6.8\}\left(\times 10^{-3}\right)$, respectively.

The vertical shear of the vertical velocity $\partial w / \partial z$ (Fig. 23c), computed from (12), shows that the agesotrophic confluence dominates over the ageostrophic speed acceleration along the axis dipole, while away from the axis the opposite occurs. As expected, the pattern of $\partial w / \partial z$ (Fig. 23c) is similar to the pattern of $w$ (Fig. 14b).

\section{2) CASES $\kappa_{d} \cong 0$ AND $\kappa_{d}>0$}

Similar to the reference case, the ageostrophic velocity $\mathbf{u}_{h}^{\prime}$ for $\kappa_{d} \cong 0$ and $\kappa_{d}>0$ (Figs. 24 and 25, respectively) has an eightlike pattern with clockwise circulation in both the cyclone (subgeostrophic) and anticyclone (supergeostrophic), again with $u_{h}^{\prime}$ larger in the anticyclone (Fig. 21). The ageostrophic speed $u_{h}^{\prime}$ in the case $\kappa_{d} \cong 0$ reaches 0.25 (cyclone) and 0.35 (anticyclone) (Fig. 24), while in the case $\kappa_{d}>0$ reaches [0.30, 0.35, 


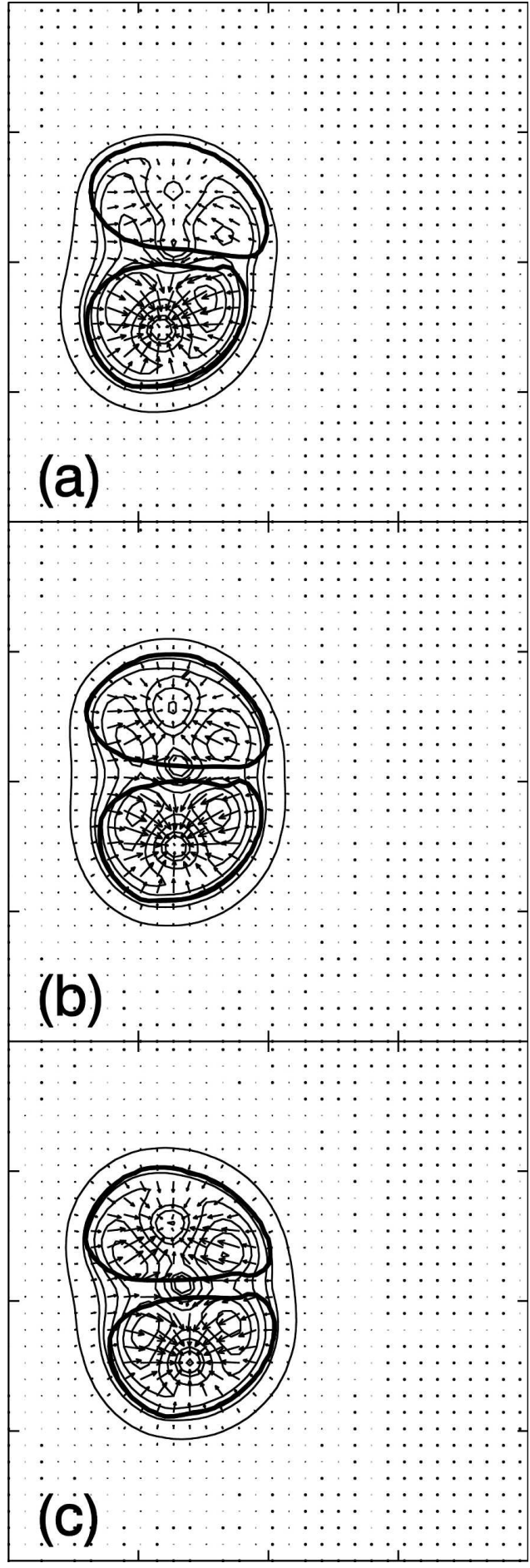

FIG. 22. Horizontal distribution of $\mathbf{u}_{h} \cdot \nabla_{h} \mathbf{u}_{h}$ at time $t=6$ i.p. for the cases (a) $\kappa_{d}<0$, (b) $\kappa_{d} \cong 0$, and (c) $\kappa_{d}>0$. Horizontal domain is $x, y \in[-\pi, \pi] c$. Only every other vector has been plotted. The magnitude $\left|\mathbf{u}_{h} \cdot \nabla_{h} \mathbf{u}_{h}\right|$ is shown (thin line, $\Delta=4 \times 10^{-3}$, with a minimum contour $2 \times 10^{-3}$ ). The contour of $\varpi_{0}= \pm 5 \times 10^{-2}$ at $z=0$ (thickest line) is included.

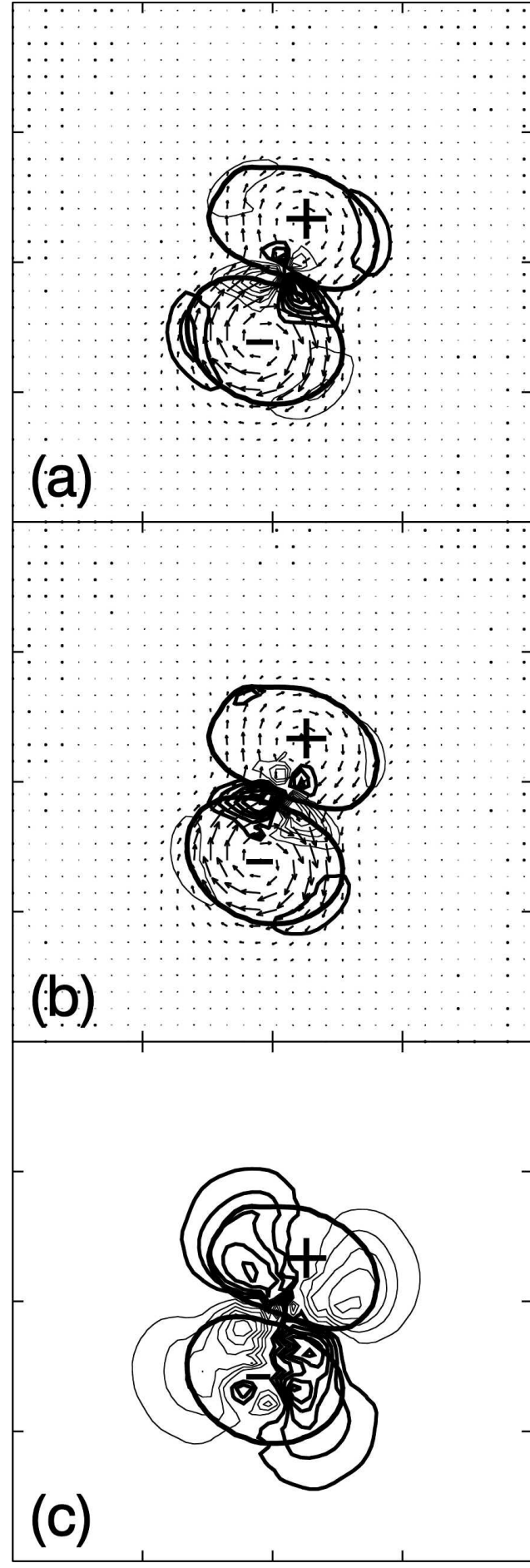

FIG. 23. Horizontal distribution of (a) $\mathbf{s}^{\prime} \cdot \nabla_{h} u_{h}^{\prime}$, (b) $u_{h}^{\prime} \boldsymbol{\nabla}_{h} \cdot \mathbf{s}^{\prime}$ $\left(\Delta= \pm 1 \times 10^{-3}\right.$ with extreme contours $\left.\pm 1 \times 10^{-3}\right)$, and (c) $\partial w / \partial z$ $\left(\Delta= \pm 1 \times 10^{-4}\right.$ with extreme contours $\left.\pm 1 \times 10^{-4}\right)$, at $t=12$ i.p. on the middle plane $(z=0)$ for the case $\kappa_{d}<0$. Horizontal domain is $x, y \in[-\pi, \pi]$. Thick (thin) contours mean positive (negative) values. The vortex locations and the ageostrophic horizontal velocity are included. 


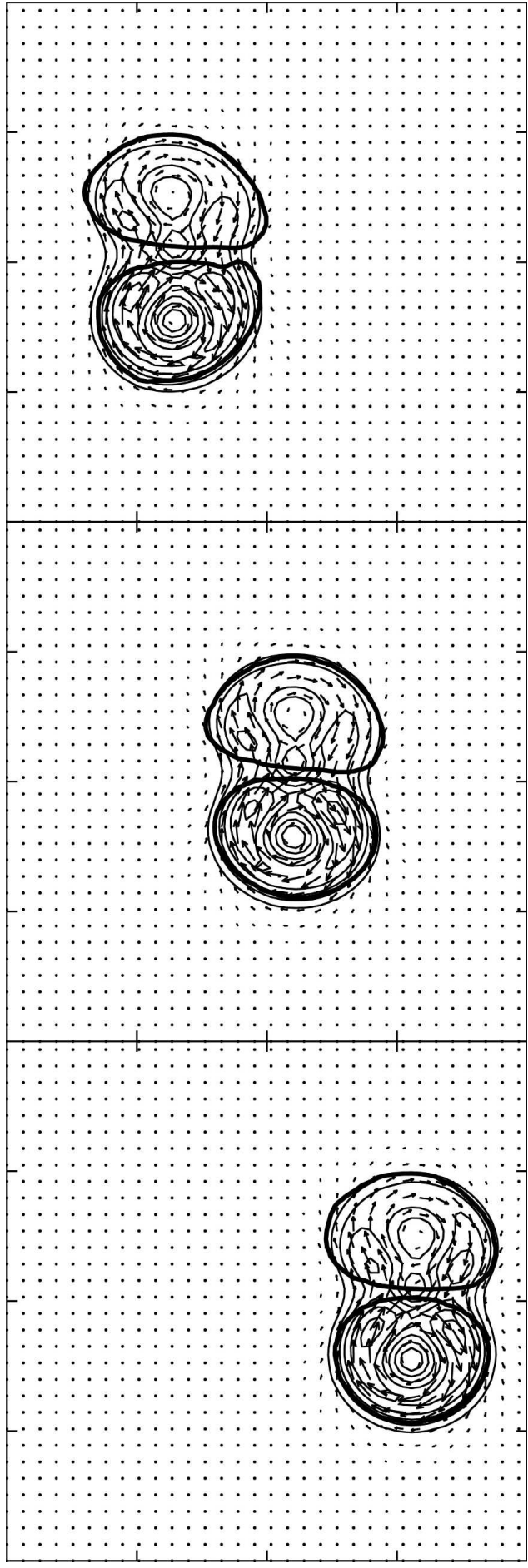

FIG. 24. As in Fig. 20 but for the case $\kappa_{d} \cong 0$.

0.30] (cyclone) and 0.35 (anticyclone) (Fig. 25) at times $t=\{6,12,18\}$ i.p. Similar to the reference simulation, the extreme values of $u_{h}^{\prime}$ occur where the horizontal advective acceleration, or more precisely the centripetal acceleration, is large (Figs. 22b,c).

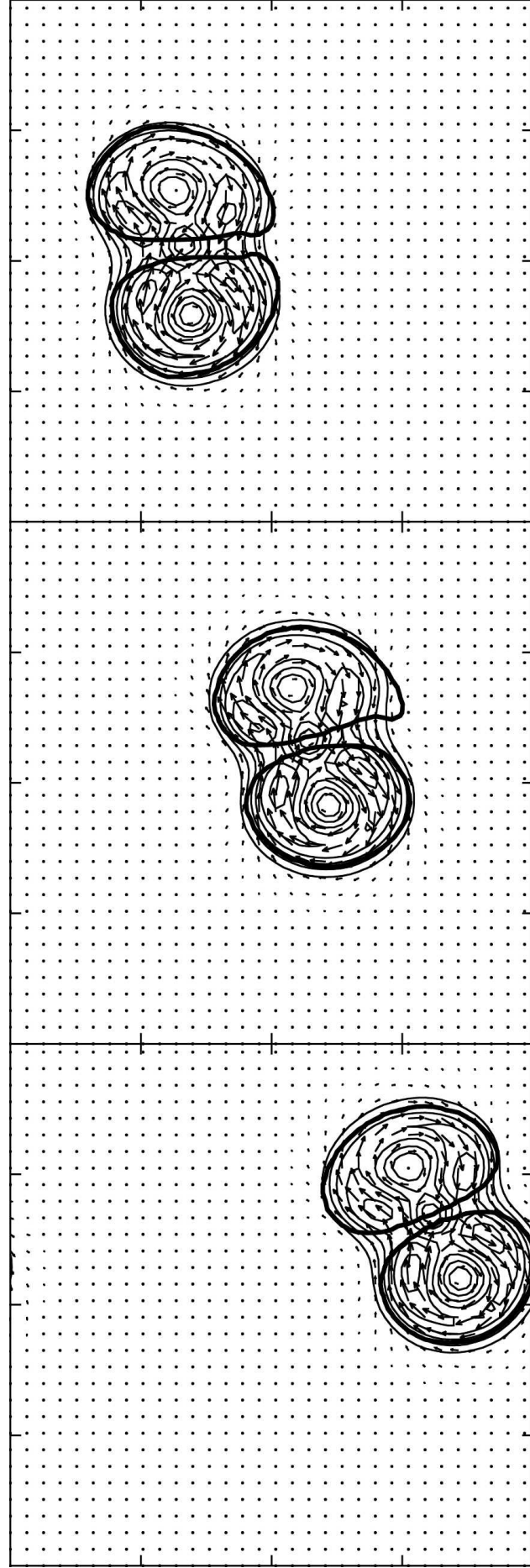

FIG. 25. As in Fig. 20 but for the case $\kappa_{d}>0$.

The minimum of ageostrophy at the dipole center, also seen in the reference simulation, is highlighted in these cases, with values of 0.10 at times $t=\{6,12,18\}$ i.p., because the cyclone is more ageotrophic than in the reference simulation. Thus, geostrophy is large in the 


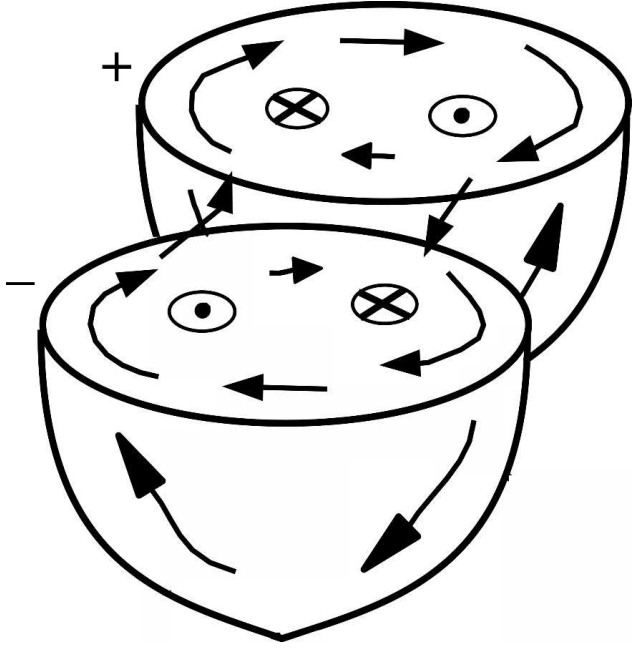

FIG. 26. Three-dimensional sketch of the ageostrophic motion in the baroclinic dipole. The circled cross and circled dot indicate upwelling and downwelling, respectively.

dipole center (seen as a minimum in $u_{h}^{\prime}$ in Figs. 24 and 25 ) because of the lack of curvature and speed acceleration at the center of the dipole axis (Figs. 22b,c).

A 3D sketch of the ageostrophic flow is shown in Fig. 26. The eightlike pattern of the horizontal ageostrophic motion and the four-cell pattern of the vertical motion are the main characteristics of the ageostrophic flow in the $3 \mathrm{D}$ vortex dipole.

\section{h. Internal oscillations}

Results from several numerical simulations starting from different values of the initial distance between vortices $d_{0}$ showed that the process of dipole formation from two ellipsoidal vortices of opposite PV highly depends on $d_{0}$. This is consistent with previous results in stratified nonrotating fluids (e.g., Couder and Basdevant 1986; Beckers et al. 2002). The dipole formation process involves the interaction between the oppositely signed PV vortices whose distance of separation $d(t)$ oscillates with time. We have found no evidence of reaching a total steady state during the first $t=150$ i.p. Equilibrium states have been found in the $2 \mathrm{D}$ case (Pierrehumbert 1980; Dritschel 1995; Billant et al. 1999); however, 3D effects and stratification may play an important role in this case.

Figure 27 shows the time series of the distance $d(t)$ during 50 i.p. For small initial distances $d_{0}$, the oscillations are small. For large $d_{0}$, the dipole experiences longer phase rotations and consequently larger oscillations. When the vortices are close enough, the phase rotation is blocked and only small internal oscillations are observed. These oscillations remain, however, long,

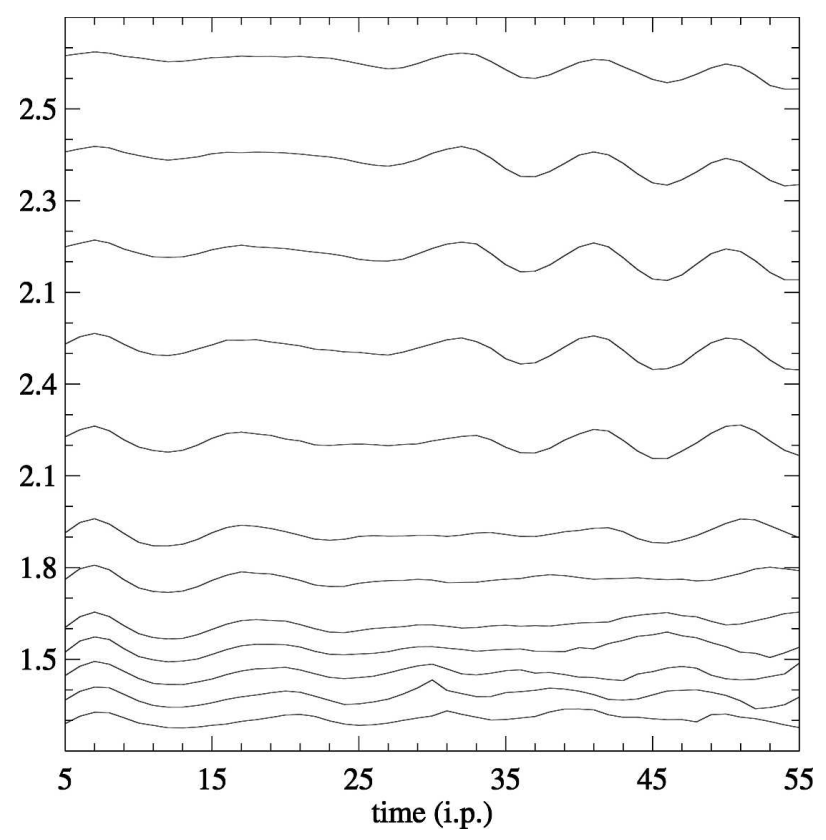

FIG. 27. Time series (from $t=5$ to 55 i.p.) of the distance between the vortices $d(t)$ as a function of the initial distance $d_{0}$.

at least longer than $t=150$ i.p. in this simulation. The time average of the dipole speed smoothly decreases with increasing $d_{0}$ (Fig. 28), and the vertical velocity $w$ is highly dependent on the initial distance $d_{0}$ (Fig. 29), decreasing with larger $d_{0}$ as well. During the dipole stabilization the direction of $\mathbf{V}_{d}$ oscillates with a period of about 6 i.p. (Fig. 7), consistent with the formation of dipoles from two interacting monopoles in stratified nonrotating fluids (Beckers et al. 2002).

This adjustment process especially affects the mesoscale vertical velocity because of its small amplitude, as observed in the time series of $\max \{w\}$, (Fig. 16), that shows periodic oscillations with a period of about 6 i.p. in both vortices, but with larger amplitude in the anticyclone. These oscillations may be important enough to change the quadrupolar pattern of the dipole vertical velocity shown in section $3 \mathrm{~d}$. The oscillations affect the horizontal ageostrophic speed $u_{h}^{\prime}$ as well (Fig. 21); however, their amplitude represent only $1 / 40$ of $u_{h}^{\prime}$, while the relative amplitude of the oscillations in the vertical velocity is 10 times larger.

To asses the effect of the shape of the initial PV configuration on the ageostrophic motion, a new simulation with two equal spheroids of oppositely signed PV anomaly was performed (Fig. 30a). In this case, the ageostrophic motion develops also a quadrupolar pattern on the mesoscale vertical velocity (Fig. 30b) and an eightlike pattern on the horizontal ageostrophic velocity (Fig. 30c). Thus, the main characteristics of the ageo- 


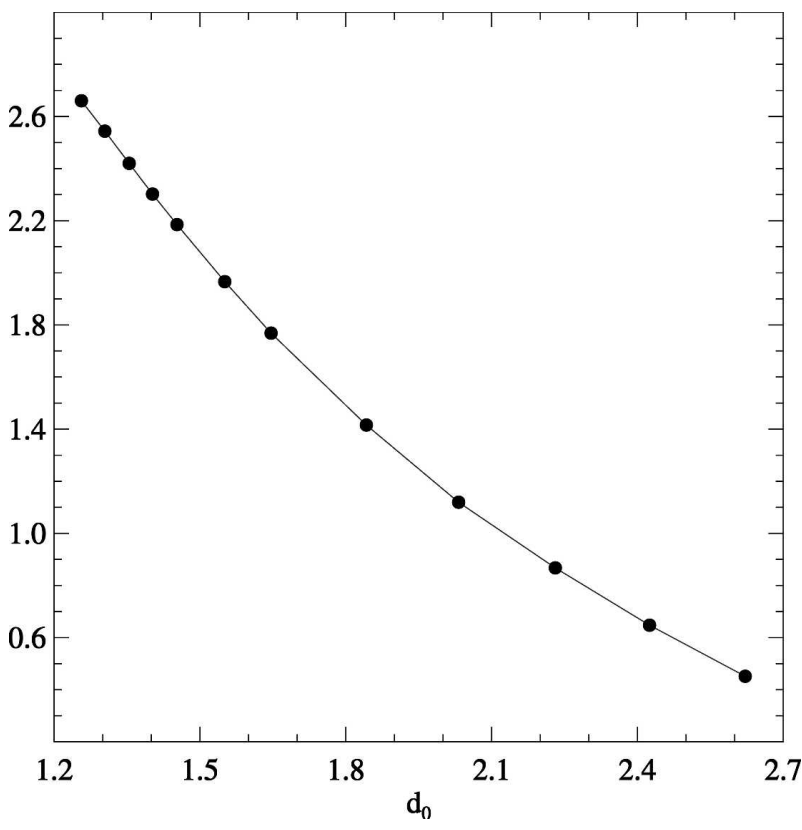

FIG. 28. The time-averaged dipole speed $\overline{\left|\mathbf{V}_{d}\right|}$ (in units of $10^{-3}$ ) as a function of the distance between the vortices $d_{0}$ in the case $\kappa_{d}<0$. The time average is from $t=5$ to 55 i.p.

strophic motion highly depend on the initial distance $d_{0}$ between vortices but are largely independent of the initial PV configuration of the vortices in the dipole.

\section{Concluding remarks}

This paper is a first attempt to describe the characteristic features of the three-dimensional ageostrophic motion in baroclinic oceanic mesoscale dipoles. Three classes of dipole motion, depending on the initial potential vorticity configuration on isopycnals in every ellipsoidal vortex, have been numerically simulated. These three cases correspond to dipole motion with negative, close to zero, and positive trajectory curvature.

The flow characteristics strongly depend on the distance between the ellipsoidal vortices $d_{0}$ relative to the vortex radius. For small $d_{0}$ the phase rotation of each vortex is blocked, the vortices move steadily as a compact dipole, that is, without internal changes, around the center of curvature. In this case the vertical velocity $w$ has an octupolar three-dimensional pattern (quadrupolar in the horizontal plane). Since the $w$ of one isolated elliptical vortex is also octupolar, the octupolar pattern of $w$ in the dipole is a consequence of the interaction between the vortices. The horizontal ageostrophic velocity of the dipole is due to the advective acceleration of the flow, particularly to the horizontal

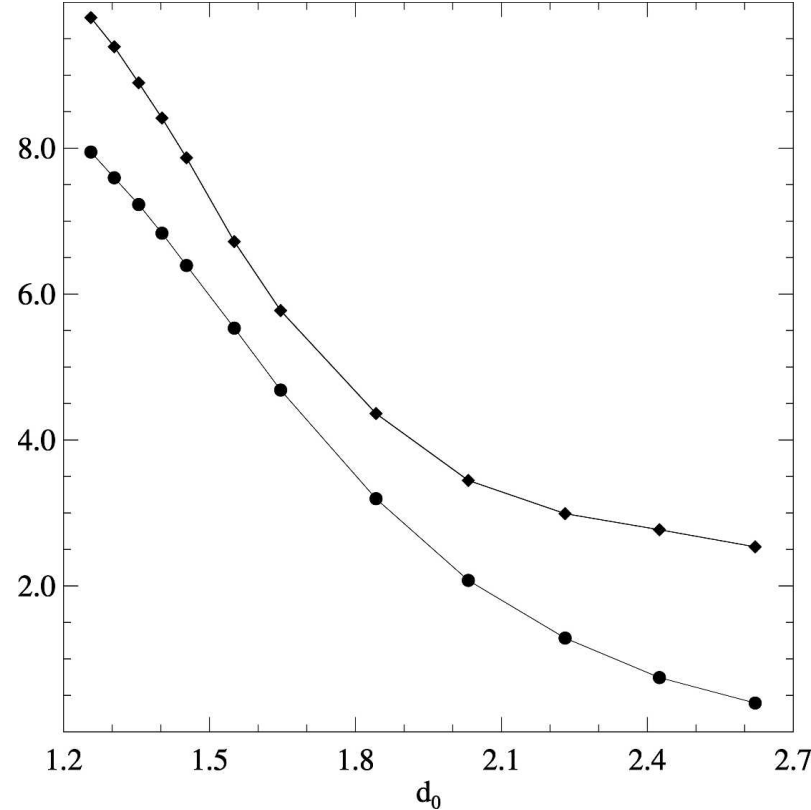

FIG. 29. The time and spatial averaged positive vertical velocity in the cyclone $\overline{\left\langle w^{+}>0\right\rangle}$ (solid dots), and anticyclone $\overline{\left\langle w^{-}>0\right\rangle}$ (solid diamonds), as a function of $d_{0}$ for the case $\kappa<0$. The time average is from $t=5$ to 55 i.p. The vertical axis is in units of $10^{-5}$.

centripetal acceleration. Since the anticyclone is supergeostrophic and the cyclone is subgeostrophic, the streamlines of the horizontal ageostrophic velocity in the dipole form an eightlike pattern, flowing along the current in the anticyclone and countercurrent in the cyclone. The speed acceleration is only relatively important at the rear and front parts of the dipole axis where the flow curvature is small, but where the flow confluence and diffluence are, respectively, very large. The geostrophy is maximal at the dipole center, on the dipole axis, where both curvature and speed acceleration are minimal.

As the distance between vortices $d_{0}$ increases, the dipole self-propagating velocity decreases, the extreme values of $|w|$ decrease, and the permissible phase rotations of every ellipsoidal vortex highly distort the octupolar pattern of $w$, which is the velocity component more susceptible to the vortex oscillations. The oscillations induce a more complex $w$ pattern composed by additional upwelling and downwelling cells. For very large $d_{0}$, the double octupolar pattern of $w$ corresponding to two separated ellipsoidal vortices is recovered, though both vortices still move slowly as a noncompact dipole around the center of curvature. In all cases, as is typical of balanced mesoscale geophysical flows, the vertical velocity is related to the advection of vertical vorticity by the horizontal shear velocity $\mathbf{u}_{h z} \cdot \nabla_{h} \zeta$.

The characteristics of the ageostrophic motion of a 


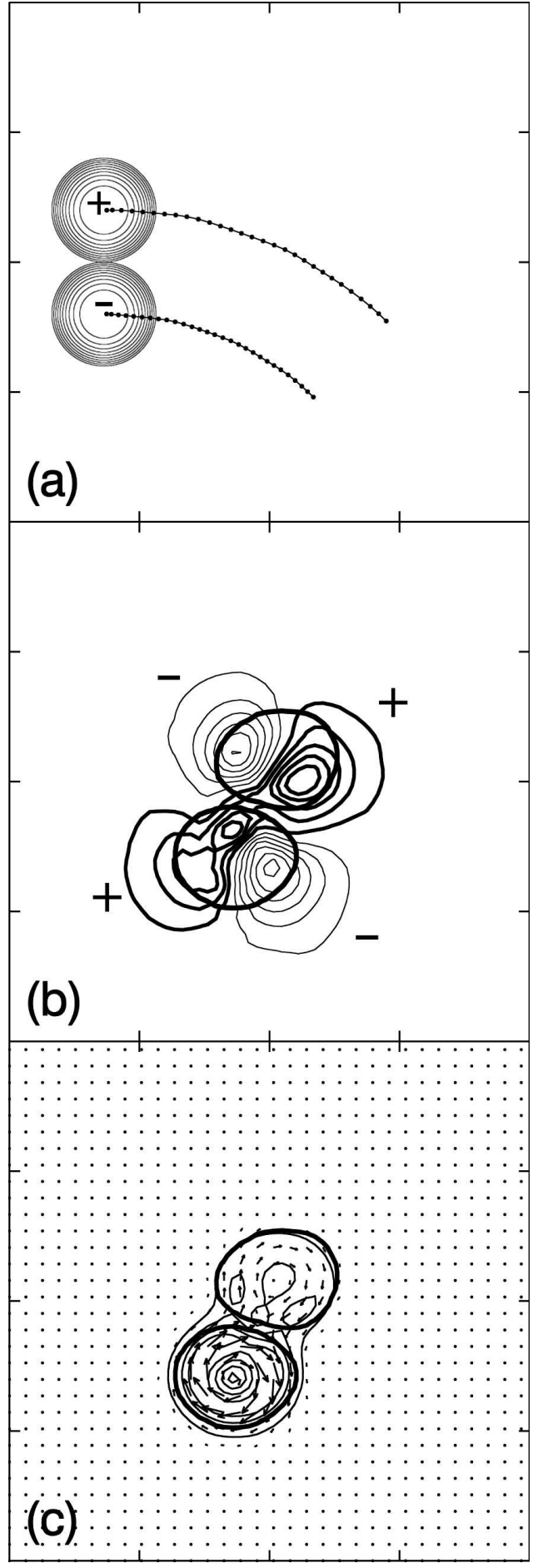

FIG. 30. Numerical simulation initialized with two equal spheroids $\left(a_{M} / c=a_{m} / c=a_{Z}=\pi / 5\right)$ for the case $\kappa<0$. Horizontal domain is $x, y \in[-\pi, \pi]$. (a) Initial PV contours in the middle isopycnal $\ell=32$. The vortex trajectory is included. (b) Horizontal distribution of $w$ at time $t=18$ i.p. on the plane $i_{Z}=27$ ( $w>0$, medium thick lines; $w$ $<0$, thin lines; $\Delta= \pm 1 \times 10^{-5}$ with extreme contours $\pm 1.5 \times 10^{-5}$ ). The contour of $\varpi_{0}= \pm 5 \times 10^{-2}$ at $z=0$ (thick line) is included. (c) Horizontal distribution of $\mathbf{u}_{h}^{\prime}$ at time $t=18$ i.p. and $z=0$ (speed $\left|\mathbf{u}_{h}^{\prime}\right|$, thin line with $\Delta=0.5 \times 10^{-1}$ and minimum contour $\left.0.25 \times 10^{-1}\right)$. Only every other vector is plotted. The contour of $\varpi_{0}= \pm 5 \times 10^{-2}$ at $z=0$ (thick line) is included. single dipole investigated here are the basis for future research on the interaction processes between dipoles, specifically dipole collision and the possible generation of inertia-gravity waves.

Acknowledgments. We acknowledge FPU Grant AP2002-1895 and a research grant from the Spanish Ministerio de Ciencia y Tecnología (REN2002-01343). We thank also two anonymous reviewers for their useful comments.

\section{APPENDIX}

\section{Derivation of (9)}

We consider the horizontal motion of a rotating fluid in constant solid body rotation with frequency $\omega$ relative to a point $\mathbf{P}(t) \equiv \mathbf{P}_{0} e^{\mathrm{i} \Omega t}$, which is itself constantly rotating around the origin of coordinates with frequency $\Omega$. We use here the boldface type to denote complex numbers as horizontal vectors in the plane $(x$, $y$ ). The motion is described by the position of every fluid particle,

$$
\mathbf{r}(\mathbf{X}, t)=\mathbf{P}_{0} e^{i \Omega t}+\left(\mathbf{X}-\mathbf{P}_{0}\right) e^{\mathrm{i} \omega t},
$$

where $\mathbf{X}$ is the label of the fluid particle (equal to its position at $t=0$ ).

The inverse function gives the particle at every location:

$$
\mathbf{R}(\mathbf{x}, t)=\mathbf{x} e^{-\mathrm{i} \omega t}-\mathbf{P}_{0} e^{\mathrm{i}(\Omega-\omega) t}+\mathbf{P}_{0} .
$$

The fluid velocity in the material description is

$$
\tilde{\mathbf{u}}(\mathbf{X}, t)=\frac{\partial r}{\partial t}(\mathbf{X}, t)=i \mathbf{P}_{0} \Omega e^{\mathrm{i} \Omega t}+i\left(\mathbf{X}-\mathbf{P}_{0}\right) \omega e^{i \omega t},
$$

Using (A2), the velocity in the spatial description is

$$
\mathbf{u}(\mathbf{x}, t)=i \mathbf{P}_{0}(\Omega-\omega) e^{i \Omega t}+i \mathbf{x} \omega .
$$

The first term above is the velocity due to the rotation of $\mathbf{P}(t)$ (which only depends on $t$ ) and the second term is the velocity due to the solid-body rotation (which only depends on $\mathbf{x})$. At points $\mathbf{x}=\chi \mathbf{P}(t)$, where $0<\chi \leq$ 1 , the ratio between the advective and local accelerations is

$$
\begin{aligned}
\frac{\left|\mathbf{u} \cdot \nabla_{h} \mathbf{u}\right|}{|\partial \mathbf{u} / \partial t|} & =\frac{\left|\omega(\Omega-\omega) \mathbf{P}(t)+\omega^{2} \mathbf{x}\right|}{|\mathbf{P}(t) \Omega(\Omega-\omega)|} \\
& =\frac{\left|\omega(\Omega-\omega)+\omega^{2} \chi\right|}{|\Omega(\Omega-\omega)|} \\
& =\frac{|\omega|}{|\Omega|} \frac{|\Omega-\omega(1-\chi)|}{|\Omega-\omega|} \gg 1
\end{aligned}
$$

for $(1-\chi)|\omega| \gg|\Omega|$. 


\section{REFERENCES}

Afanasyev, Y., 2003: Spontaneous emission of gravity waves by interacting vortex dipoles in a stratified fluid: Laboratory experiments. Geophys. Astrophys. Fluid Dyn., 97, 79-95.

Ahlnäs, K., T. C. Royer, and T. H. George, 1987: Multiple dipole eddies in the Alaska Coastal Current detected with Landsat Thematic Mapper Data. J. Geophys. Res., 92, 13 041-13 047.

Batchelor, G. K., 1967: An Introduction to Fluid Mechanics. Cambridge University Press, 615 pp.

Beckers, M., H. J. H. Clercx, G. J. F. van Heijst, and R. Verzicco, 2002: Dipole formation by two interacting shielded monopoles in a stratified fluid. Phys. Fluids, 14, 704-720.

Billant, P., P. Brancher, and J.-M. Chomaz, 1999: Threedimensional stability of a vortex pair. Phys. Fluids, 11, 20692077.

Bush, A. B. G., J. C. McWilliams, and W. R. Peltier, 1996: The formation of oceanic eddies in symmetric and asymmetric jets. Part II: Late time evolution and coherent vortex formation. J. Phys. Oceanogr., 26, 1825-1848.

Couder, Y., and C. Basdevant, 1986: Experimental and numerical study of vortex couples in two-dimensional flows. J. Fluid Mech., 173, 225-251.

Daley, R., 1991: Atmospheric Data Analysis. Cambridge University Press, $457 \mathrm{pp}$

de Ruijter, W. P. M., H. M. van Aken, E. J. Beier, J. R. E. Lutjeharms, R. P. Matano, and M. W. Schouten, 2004: Eddies and dipoles around South Madagascar: Formation, pathways and large-scale impact. Deep-Sea Res. I, 51, 383-400.

Dritschel, D. G., 1995: A general theory for two-dimensional vortex interactions. J. Fluid Mech., 293, 269-303.

- and M. H. P. Ambaum, 1997: A contour-advective semiLagrangian algorithm for the simulation of fine-scale conservative fields. Quart. J. Roy. Meteor. Soc., 123, 1097-1130.

— rotating stably-stratified geophysical flows. J. Fluid Mech., 488, $123-150$.

Eames, I., and J. B. Flór, 1998: Fluid transport by dipolar vortices. Dyn. Atmos. Oceans, 28, 93-105.

Fedorov, K. N., and A. I. Ginsburg, 1986: "Mushroom-like" currents (vortex dipoles) in the ocean and in a laboratory tank. Ann. Geophys., 4, 507-516.

Flierl, G. R., 1987: Isolated eddy models in geophysics. Аnпи. Rev. Fluid Mech., 19, 493-530.

— meandering. J. Mar. Res., 51, 529-560.

_ , V. D. Larichev, J. C. McWilliams, and G. M. Reznik, 1980: The dynamics of baroclinic and barotropic solitary eddies. Dyn. Atmos. Oceans, 5, 1-41.

—, M. E. Stern, and J. A. Whitehead, 1983: The physical significance of modons-Laboratory experiments and general integral constraints. Dyn. Atmos. Oceans, 7, 233-263.

—, P. Malanotte-Rizzoli, and N. J. Zabusky, 1987: Nonlinear waves and coherent vortex structures in barotropic $\beta$-plane jets. J. Phys. Oceanogr., 17, 1408-1438.

Flór, J. B., and G. J. F. van Heijst, 1994: An experimental study of dipolar vortex structures in a stratified fluid. J. Fluid Mech., 279, 101-133.

Ford, R., M. E. McIntyre, and W. A. Norton, 2000: Balance and the slow quasimanifold: Some explicit results. J. Atmos. Sci., 57, 1236-1254

Fuentes, O. U. V., and G. J. F. van Heijst, 1994: Experimental study of dipolar vortices on a topographic beta-plane. J. Fluid Mech., 259, 79-106.

,-- , and B. E. Cremers, 1995: Chaotic transport by dipolar vortices on a beta-plane. J. Fluid Mech., 291, 139-161.

Gill, A. E., 1982: Atmosphere-Ocean Dynamics., Academic Press, $662 \mathrm{pp}$.

Ginzburg, A. I., and K. N. Fedorov, 1984: The evolution of a mushroom-formed current in the ocean. Dokl. Akad. Nauk SSSR, 274, 481-484.

Griffiths, R. W., and E. J. Hopfinger, 1987: Coalescing of geostrophic vortices. J. Fluid Mech., 178, 73-97.

Gryanik, V. M., T. N. Doronina, D. J. Olbers, and T. H. Warncke, 2000: The theory of three-dimensional hetons and vortexdominated spreading in localized turbulent convection in a fast rotating stratified fluid. J. Fluid Mech., 423, 71-125.

Hesthaven, J. S., J. P. Lynov, A. H. Nielsen, J. J. Rasmussen, M. R. Schmidt, E. G. Shapiro, and S. K. Turitsyn, 1995: Dynamics of a nonlinear dipole vortex. Phys. Fluids, 7, 2220 2229

Hogg, N. G., and H. M. Stommel, 1985: The heton, an elementary interaction between discrete baroclinic geostrophic vortices, and its implications concerning eddy heatflow. Proc. Roy. Soc. London, 397A, 1-20.

Hoskins, B. J., M. E. McIntyre, and A. W. Robertson, 1985: On the use and significance of isenotropic potential-vorticity maps. Quart. J. Roy. Meteor. Soc., 111, 877-946.

Ikeda, M., L. A. Mysak, and W. J. Emery, 1984: Observation and modeling of satellite-sensed meanders and eddies off Vancouver Island. J. Phys. Oceanogr., 14, 3-21.

Johannessen, J. A., E. Svendsen, O. M. Johannessen, and K. Lygre, 1989: Three-dimensional structure of mesoscale eddies in the Norwegian Coastal Current. J. Phys. Oceanogr., 19, 3-19.

Khvoles, R., D. Berson, and Z. Kizner, 2005: The structure and evolution of elliptical barotropic modons. J. Fluid Mech., 530, $1-30$.

Kizner, Z., 1997: Solitary Rossby waves with baroclinic modes. $J$. Mar. Res., 55, 671-685.

— D. Berson, and R. Khvoles, 2003: Non-circular baroclinic beta-plane modons: Constructing stationary solutions. $J$. Fluid Mech., 489, 199-228.

Kloosterziel, R. C., and G. J. F. van Heijst, 1991: An experimental study of unstable barotropic vortices in a rotating fluid. $J$. Fluid Mech., 223, 1-24.

Lamb, H., 1932: Hydrodynamics. 6th ed. Cambridge University Press, $738 \mathrm{pp}$.

McGillicuddy, D. J., Jr., and Coauthors, 1998: Influence of mesoscale eddies on new production in the Sargasso Sea. Nature, 394, 263-266.

McWilliams, J. C., 1983: Interactions of isolated vortices. II: Modon generation by monopole collision. Geophys. Astrophys. Fluid Dyn., 19, 207-227.

Meleshko, V. V., and G. J. F. van Heijst, 1994: On Chaplygin investigations of two dimensional vortex structures in an invisquid fluid. J. Fluid Mech., 272, 157-182.

Millot, C., 1985: Some features of the Algerian Current. J. Geophys. Res., 90, 7169-7176.

Mohebalhojeh, A. R., and D. G. Dritschel, 2000: On the representation of gravity waves in numerical models of the shallow water equations. Quart. J. Roy. Meteor. Soc., 126, 669-688.

— and - 2001: Hierarchies of balance conditions for the $f$-plane shallow water equations. J. Atmos. Sci., 58, 24112426. 
Morel, Y., and J. C. McWilliams, 1997: Evolution of isolated interior vortices in the ocean. J. Phys. Oceanogr., 27, 727-748.

Orlandi, P., and G. F. Carnevale, 1999: Evolution of isolated vortices in a rotating fluid of finite depth. J. Fluid Mech., 381, 239-269.

Pallàs-Sanz, E., and A. Viúdez, 2005: Diagnosing mesoscale vertical velocity from horizontal velocity and density data. $J$. Phys. Oceanogr., 35, 1744-1762.

Pedlosky, J., 1985: Instability of heton clouds. J. Atmos. Sci., 42, 1477-1486.

Pierrehumbert, R. T., 1980: A family of steady, translating vortex pairs with distributed vorticity. J. Fluid Mech., 99, 129-144.

Praud, O., and A. M. Fincham, 2005: The structure and dynamics of dipolar vortices in a stratified fluid. J. Fluid Mech., 544, $1-22$.

Robinson, A. R., M. A. Spall, and N. Pinardi, 1988: Gulf Stream simulations and the dynamics of ring and meander processes. J. Phys. Oceanogr., 18, 1811-1853.

— ties during the 1989 JGOFS bloom study. Deep-Sea Res. II, 40, 9-35.

Sansón, L. Z., G. J. F. van Heijst, and N. A. Backx, 2001: Ekman decay of a dipolar vortex in a rotating fluid. Phys. Fluids, 13, $440-451$.

Shimada, K., and A. Kubokawa, 1997: Nonlinear evolution of linearly unstable barotropic boundary currents. J. Phys. Oceanogr., 27, 1326-1348.

Spall, M. A., 1995: Frontogenesis, subduction, and cross-front exchange at upper ocean fronts. J. Geophys. Res., 100, 25432557.
— , and A. R. Robinson, 1990: Regional primitive equation studies of the Gulf Stream meander and ring formation region. J. Phys. Oceanogr., 20, 985-1016.

Stern, M. E., 1975: Minimal properties of planetary eddies. J. Mar. Res., 40, 57-74.

Strass, V. H., 1992: Chlorophyll patchiness caused by mesoscale upwelling at fronts. Deep-Sea Res. I, 39, 75-96.

Vandermeirsch, F. O., X. J. Carton, and Y. G. Morel, 2003a: Interaction between an eddy and a zonal jet. Part I. One-anda-half-layer model. Dyn. Atmos. Oceans, 36, 247-270.

$\_, \ldots$, and — 2003b: Interaction between an eddy and a zonal jet. Part II. Two-and-a-half-layer model. Dyn. Atmos. Oceans, 36, 271-296.

van Heijst, G. J. F., and J. B. Flór, 1989: Dipole formation and collisions in a stratified fluid. Nature, 340, 212-215.

Viúdez, A., and D. G. Dritschel, 2003: Vertical velocity in mesoscale geophysical flows. J. Fluid Mech., 483, 199-223.

— , and — 2004a: Optimal potential vorticity balance of geophysical flows. J. Fluid Mech., 521, 343-352.

- , and $-2004 \mathrm{~b}$ : Potential vorticity and the quasigeostrophic and semigeostrophic mesoscale vertical velocity. $J$. Phys. Oceanogr., 34, 865-887.

Voropayev, S. I., Y. D. Afanasyev, and I. A. Filippov, 1991: Horizontal jets and vortex dipoles in a stratified fluid. J. Fluid Mech., 227, 543-566.

—, X. Z. Zhang, D. L. Boyer, H. J. S. Fernando, and P. C. Wu, 1997: Horizontal jets in a rotating stratified fluid. Phys. Flu$i d s, \mathbf{9}, 115-126$. 